\title{
CENTER PROBLEM FOR $\Lambda-\Omega$ DIFFERENTIAL SYSTEMS
}

\author{
JAUME LLIBRE ${ }^{1}$, RAFAEL RAMÍREZ² AND VALENTÍN RAMÍREZ ${ }^{1}$
}

Abstract. The $\Lambda-\Omega$ systems are the real planar polynomial differential equations of degree $m$

$$
\dot{x}=-y(1+\Lambda)+x \Omega, \quad \dot{y}=x(1+\Lambda)+y \Omega,
$$

where $\Lambda=\Lambda(x, y)$ and $\Omega=\Omega(x, y)$ are polynomials of degree at most $m-1$ such that $\Lambda(0,0)=\Omega(0,0)=0$. We study the center problem for these $\Lambda-\Omega$ systems. Any planar vector fields with linear type center can be written as an $\Lambda-\Omega$ systems if and only if the Poincaré-Liapunov first integral is of the form $F=\frac{1}{2}\left(x^{2}+y^{2}\right)(1+O(x, y))$. These kind of linear type centers are called weak centers, they contain the class of center studied by Alwash and Lloyd [1], and also contain the uniform isochronous centers, and the holomorphic isochronous centers, but they do not coincide with the all class of isochronous centers.

The main objective of this paper is to study the center problem for two particular classes of $\Lambda-\Omega$ systems of degree $m$.

First if $\Lambda=\mu\left(a_{2} x-a_{1} y\right)$, and $\Omega=a_{1} x+a_{2} y+\Omega_{m-1}$, where $\mu, a_{1}, a_{2}$ are constants and $\Omega_{m-1}=\Omega_{m-1}(x, y)$ is a homogenous polynomial of degree $m-1$, then we prove the following results.

(i) These $\Lambda-\Omega$ systems have a weak center at the origin if and only if $(\mu+$ $m-2)\left(a_{1}^{2}+a_{2}^{2}\right)=0$, and $\int_{0}^{2 \pi} \Omega_{m-1}(\cos t, \sin t) d t=0 ;$

(ii) If $m=2,3,4,5,6$ and $(\mu+m-2)\left(a_{1}^{2}+a_{2}^{2}\right) \neq 0$, then the given $\Lambda-\Omega$ systems have a weak center at the origin if and only if these systems after a linear change of variables $(x, y) \longrightarrow(X, Y)$ are invariant under the transformations $(X, Y, t) \longrightarrow(-X, Y,-t)$. .

Second if $\Lambda=a_{1} x+a_{2} y$, and $\Omega=\Omega_{m-1}$, where $a_{1}, a_{2}$ are constants and $\Omega_{m-1}=\Omega_{m-1}(x, y)$ is a homogenous polynomial of degree $m-1$, then we prove the following results.

(i) These $\Lambda-\Omega$ systems have a weak center at the origin if and only if $a_{1}=$ $a_{2}=0$, and $\int_{0}^{2 \pi} \Omega_{m-1}(\cos t, \sin t) d t=0$

(ii) If $m=2,3,4,5$ and $a_{1}^{2}+a_{2}^{2} \neq 0$, then the given $\Lambda-\Omega$ systems have a weak center at the origin if and only if these systems after a linear change of variables $(x, y) \longrightarrow(X, Y)$ are invariant under the transformations $(X, Y, t) \longrightarrow(-X, Y,-t)$.

We observe that the main difficulty to prove results (ii) for $m>6$ is related with the huge computations necessary for proving them.

2010 Mathematics Subject Classification. 34C05, 34C07.

Key words and phrases. linear type center, analytic planar differential system, weak center, reversible system. 


\section{INTRODUCTION}

Let $\mathcal{X}=P \frac{\partial}{\partial x}+Q \frac{\partial}{\partial y}$ be the real planar polynomial vector field associated to the real planar polynomial differential system

$$
\dot{x}=P(x, y), \quad \dot{y}=Q(x, y),
$$

where the dot denotes derivative with respect to an independent variables here called the time $t$, and $P$ and $Q$ are real coprime polynomials in $\mathbb{R}[x, y]$. We say that polynomial differential system (1) has degree $m=\max \{\operatorname{deg} P, \operatorname{deg} Q\}$.

In what follows we assume that the origin $O:=(0,0)$ is a singular or equilibrium point, i.e. $P(0,0)=Q(0,0)=0$.

The equilibrium point $O$ is a center if there exists an open neighborhood $U$ of $O$ where all the orbits contained in $U \backslash\{O\}$ are periodic.

Suppose that the polynomial differential system (1) has a center at the origin. It known that doing a linear change of variables and perhaps a constant scaling of the independent variable, system (1) becomes in one of the next three systems:

$$
\begin{array}{ll}
\dot{x}=-y+X(x, y), & \dot{y}=x+Y(x, y), \\
\dot{x}=y+X(x, y), & \dot{y}=Y(x, y), \\
\dot{x}=X(x, y), & \dot{y}=Y(x, y),
\end{array}
$$

where $X(x, y)$ and $Y(x, y)$ are polynomials starting at least with quadratic terms in a neighborhood of the origin. If the origin of system (1) is a center we say that it is of linear type, nilpotent or degenerate if after a linear change of variables and a scaling of the time it can be written as the first, second or third system of (2), respectively.

The study of the centers of analytical or polynomial differential systems (1) has a long history. The first works are due to Poincaré [15] and Dulac [6]. Later on were developed by Bendixson [3], Frommer [7], Liapunov [12] and many others.

Here we shall work with the differential systems of the form

$$
\dot{x}=-y+X, \quad \dot{y}=x+Y,
$$

where $X=X(x, y)$ and $Y=Y(x, y)$ are polynomials, and the degree of the polynomial differential system (3) is $m=\max \{\operatorname{deg} X, \operatorname{deg} Y\} \geq 2$. The center-focus problem asks about conditions on the coefficients of $X$ and $Y$ for distinguish when the origin of system (3) is either a focus or a center.

In this paper we shall study the particular case of differential systems (3) of the form

$$
\dot{x}=-y(1+\Lambda)+x \Omega, \quad \dot{y}=x(1+\Lambda)+y \Omega,
$$

where $\Lambda=\Lambda(x, y)$ and $\Omega=\Omega(x, y)$ are polynomials such $m=\max \{\operatorname{deg} \Lambda, \operatorname{deg} \Omega\}+$ 1.

Systems (4) are called $\Lambda-\Omega$ systems. First we observe that if these systems have a center at the origin then they have a first integral of the form

$$
F=\frac{1}{2}\left(x^{2}+y^{2}\right)(1+O(x, y))
$$


This kind of centers having first integral of the form (5) are called weak centers, they contain the uniform isochronous centers and the holomorphic isochronous centers (for a prof of these results see [11]), but they do not coincide with the all class of isochronous centers (see Remark 19 of [11]).

This class of planar polynomial differential systems (4) appear in different fields. If we assume that $\Lambda=\lambda\left(x^{2}+y^{2}\right)-1$, and $\Omega=\omega\left(x^{2}+y^{2}\right)$, then system (4) becomes

$$
\dot{x}=-y \lambda\left(x^{2}+y^{2}\right)+x \omega\left(x^{2}+y^{2}\right), \quad \dot{y}=x \lambda\left(x^{2}+y^{2}\right)+y \omega\left(x^{2}+y^{2}\right),
$$

which is called a lambda-omega system (see for instance [8]). On the other hand it is well known the following result (see for instance [2]).

Theorem 1. Let $\mathcal{X}$ be a polynomial vector field associated to the differential system (3).

(i) Then $\mathcal{X}$ has either a focus or a center at the origin, and under a formal change of coordinates the differential system associated to $\mathcal{X}$ can be written in the Birkhoff normal form

$$
\begin{aligned}
& \dot{x}=-y\left(1+S_{2}\left(x^{2}+y^{2}\right)\right)+x S_{1}\left(x^{2}+y^{2}\right):=P, \\
& \dot{y}=x\left(1+S_{2}\left(x^{2}+y^{2}\right)\right)+y S_{1}\left(x^{2}+y^{2}\right):=Q,
\end{aligned}
$$

where $S_{j}=S_{j}\left(x^{2}+y^{2}\right)$ for $j=1,2$ are formal series in the variable $x^{2}+y^{2}$ and such that $S_{2}(0)=S_{1}(0)=0$.

(i) If $\mathcal{X}$ has a center at the origin then the formal system (6) becomes analytic with $S_{1}\left(x^{2}+y^{2}\right)=0$ (see for instance $[15,12]$ ) with the first integral $x^{2}+y^{2}$. Consequently any linear type center locally is a weak center.

Finally, by applying the inverse approach in ordinary differential equations see [9] the following theorem is proved and shows the importance of system (4) in the theory of ordinary differential equations (see Theorem 13 in [11]).

Theorem 2. The polynomial differential system (3) has a weak center at the origin if and only if it can be written as (4) with

$$
\begin{aligned}
& \Lambda=\sum_{j=2}^{m}\left(\frac{j+1}{2} \Upsilon_{j-1}+\frac{j}{2} g_{1} \Upsilon_{j-2}+\ldots+\frac{3}{2} g_{j-2} \Upsilon_{1}+g_{j-1}\right), \\
& \Omega=\frac{1}{2} \sum_{j=2}^{m}\left(\left\{\Upsilon_{j-1}, H_{2}\right\}+g_{1}\left\{\Upsilon_{j-2}, H_{2}\right\}+\ldots+g_{j-2}\left\{\Upsilon_{1}, H_{2}\right\}\right),
\end{aligned}
$$

where $\Upsilon_{0}=1, g_{0}=1, g_{j}$ and $\Upsilon_{j}$ are homogenous polynomials of degree $j$ for $j \geq 1$ and has the first integral of the form

$$
H=H_{2} \Phi=H_{2}\left(1+\mu_{1} \Upsilon_{1}+\ldots+\mu_{m-1} \Upsilon_{m-1}\right),
$$

where $H_{2}=\left(x^{2}+y^{2}\right) / 2$, and $\mu_{j}=\mu_{j}(x, y)$ is a convenient analytic function in the neighborhood of the origin for $j=1, \ldots, m-1$.

\section{Statement of the MAin Results}

In this section we give the statements of our main results which will be proved in sections 4, 5 and 6 , also we state some conjectures. 
Proposition 3. The polynomial differential system of degree $m$

$$
\begin{aligned}
& \dot{x}=-y\left(1+(m-2)\left(a_{1} y-a_{2} x\right)\right)+x\left(a_{1} x+a_{2} y+\Omega_{m-1}\right), \\
& \left.\dot{y}=x\left(1+(m-2) a_{1} y-a_{2} x\right)\right)+y\left(a_{1} x+a_{2} y+\Omega_{m-1}\right),
\end{aligned}
$$

where $\Omega_{m-1}=\Omega_{m-1}(x, y)$ is a homogenous polynomial of degree $m-1$, has a weak center at the origin if and only if

$$
\int_{0}^{2 \pi} \Omega_{m-1}(\cos t, \sin t) d t=0 .
$$

Moreover system (7) has the first integral

$$
H=\frac{H_{2}}{\left(1+\frac{m-1}{m+1} G(x, y)+\Gamma(x, y)+\Phi\left(H_{2}\right)\right)^{2 /(m-1)}}=H_{2}(1+\text { h.o.t. }),
$$

where $G$ is a polynomial of degree $m-1$ and such that $\left\{H_{2}, G\right\}=-(m+1)\left(a_{1} x+\right.$ $\left.a_{2} y+\Omega_{m-1}\right), \Gamma=\Gamma(x, y)$ is a convenient polynomial and $\Phi\left(H_{2}\right)$ is a convenient polynomial if $m$ even and a convenient function if $m$ is odd (see the proof for the expressions of $\Gamma$ and $\Phi)$.

We observe that if we take $a_{1}=a_{2}=0$ in Proposition 3 we obtain following corollary.

Corollary 4. The polynomial differential system of degree $m$

$$
\dot{x}=-y+x \Omega_{m-1} \quad \dot{y}=x+y \Omega_{m-1},
$$

has a weak uniform center at the origin if and only if (8) holds. Moreover system (10) has the Poincaré-Liapunov first integral (9) where $G$ is a homogenous polynomial of degree $m-1$ and such that $\left\{H_{2}, G\right\}=\Omega_{m-1}$.

Conti in [5] proved the first part of Corollary4, but the second part providing an explicit expression of the first integral of system (10) is new.

Remark 5. The weak centers obtained in Proposition 3 and Corollary 4 in general are not invariant with respect to the transformation $(x, y, t) \longrightarrow(-x, y,-t)$. Indeed the polynomial differential system of degree $2 k$ given by formula $(7)$ and $(10)$, when $\Omega_{2 k-1}$ is an arbitrary polynomial of degree $2 k-1$, in general is not reversible and has the weak center at the origin.

We recall that a polynomial differential system has a uniform center at the origin if written in polar coordinates $x=r \cos \theta, y=r \sin \theta$ we obtain that $\dot{\theta}$ is constant.

Conjecture 6. The polynomial differential system of degree $m$

$$
\begin{aligned}
& \dot{x}=-y\left(1+\mu\left(a_{2} x-a_{1} y\right)\right)+x\left(a_{1} x+a_{2} y+\Omega_{m-1}\right), \\
& \dot{y}=x\left(1+\mu\left(a_{2} x-a_{1} y\right)\right)+y\left(a_{1} x+a_{2} y+\Omega_{m-1}\right),
\end{aligned}
$$

where $(\mu+m-2)\left(a_{1}^{2}+a_{2}^{2}\right) \neq 0$, and $\Omega_{m-1}=\Omega_{m-1}(x, y)$ is a homogenous polynomial of degree $m-1$ has a weak center at the origin if and only if system (11) after a linear change of variables $(x, y) \longrightarrow(X, Y)$ it is invariant under the transformations $(X, Y, t) \longrightarrow(-X, Y,-t)$.

Theorem 7. Conjecture 6 holds for $m=2,3,4,5,6$. 
Conjecture 8. The polynomial differential system of degree $m$

$$
\begin{aligned}
& \dot{x}=-y\left(1+a_{1} x+a_{2} y\right)+x \Omega_{m-1}, \\
& \dot{y}=x\left(1+a_{1} x+a_{2} y\right)+y \Omega_{m-1},
\end{aligned}
$$

where $a_{1}^{2}+a_{2}^{2} \neq 0$, and $\Omega_{m-1}=\Omega_{m-1}(x, y)$ is a homogenous polynomial of degree $m-1$ has a weak center at the origin if and only if system (12) after a linear change of variables $(x, y) \longrightarrow(X, Y)$ it is invariant under the transformations $(X, Y, t) \longrightarrow(-X, Y,-t)$.

Theorem 9. Conjecture 8 holds for $m=2,3,4,5,6$.

The only difficulty for proving Conjectures 6 and 8 for the $\Lambda-\Omega$ systems of degree $m$ with $m>6$ is the huge number of computations for obtaining the conditions that characterize the centers.

\section{PReliminary RESUlts}

In the proofs of the main results of this paper it plays a very important role the following results and notations which we can find in [11] .

As usual the Poisson bracket of the functions $f(x, y)$ and $g(x, y)$ is defined as

$$
\{f, g\}:=\frac{\partial f}{\partial x} \frac{\partial g}{\partial y}-\frac{\partial f}{\partial y} \frac{\partial g}{\partial x} .
$$

The following result is a simple consequence of the Liapunov result given in Theorem 1 , page 276 of [12] .

Corollary 10. Let $U=U(x, y)$ be a homogenous polynomial of degree $m$. The linear partial differential equation $\left\{H_{2}, V\right\}=U$, has a unique homogenous polynomial solution $V$ of degree $m$ if $m$ is odd; and if $V$ is a homogenous polynomial solution when $m$ is even then any other homogenous polynomial solution is of the form $V+c\left(x^{2}+y^{2}\right)^{m / 2}$ with $c \in \mathbb{R}$. Moreover, for $m$ even these solutions exist if and only if $\left.\int_{0}^{2 \pi} U(x, y)\right|_{x=\cos t, y=\sin t} d t=0$.

Proposition 11 (see Proposition 6 of [10]). The next relation holds

$$
\left.\int_{0}^{2 \pi}\left\{H_{2}, \Psi\right\}\right|_{x=\cos t, y=\sin t} d t=0
$$

for an arbitrary $C^{1}$ function $\Psi=\Psi(x, y)$ defined in the interval $[0,2 \pi]$.

Proposition 12 (see Proposition 24 of [10]). Consider the polynomial differential system (1) of degree $m$ which satisfies

$$
\left.\int_{0}^{2 \pi}\left(\frac{\partial P}{\partial x}+\frac{\partial Q}{\partial y}\right)\right|_{x=\cos t, y=\sin t} d t=0 .
$$

Then there exist polynomials $F=F(x, y)$ and $G=G(x, y)$ of degree $m+1$ and $m-1$ respectively such that system (1) can be written as

$$
\dot{x}=P=\{F, x\}+(1+G)\left\{H_{2}, x\right\}, \quad \dot{y}=Q=\{F, y\}+(1+G)\left\{H_{2}, y\right\},
$$

with $G(0,0)=0$. 
We need the following definitions and notions. A function $V=V(x, y)$ is an inverse integrating factor of system (1) in an open subset $U \subset \mathbb{R}^{2}$ if $V \in C^{1}(U), V \not \equiv$ 0 in $U$ and $\frac{\partial}{\partial x}\left(\frac{P}{V}\right)+\frac{\partial}{\partial y}\left(\frac{Q}{V}\right)=0$

Theorem 13 (Reeb 's criterion). (see for instance [16]) The analytic differential system $\dot{x}=-y+\sum_{j=2}^{\infty} X_{j}, \quad \dot{y}=x+\sum_{j=2}^{\infty} Y_{j}$ has a center at the origin if and only if there is a local nonzero analytic inverse integrating factor of the form $V=1+$ h.o.t. in a neighborhood of the origin.

An analytic inverse integrating factor of the form $V=1+$ h.o.t. in a neighborhood of the origin is called a Reeb inverse integrating factor.

The analytic function

$$
H=\sum_{j=2}^{\infty} H_{j}(x, y)=\frac{1}{2}\left(x^{2}+y^{2}\right)+\sum_{j=3}^{\infty} H_{j}(x, y),
$$

where $H_{j}$ is homogenous polynomials of degree $j>1$, is called the PoincaréLiapunov local first integral if $H$ is constant on the solutions of (3).

Theorem 14 (see Theorem 13 and Remark 14 of [11]). Consider the polynomial vector field $\mathcal{X}=\left(-y+\sum_{j=2}^{m} X_{j}\right) \frac{\partial}{\partial x}+\left(x+\sum_{j=2}^{m} Y_{j}\right) \frac{\partial}{\partial y}$. Then this vector field has a Poincaré-Liapunov local first integral $H$ if and only if it has a Reeb inverse integrating factor $V$. Moreover, the differential system associated to the vector field $\mathcal{X}$ for which $H=\left(x^{2}+y^{2}\right) / 2+$ h.o.t. is a local first integral can be written as

$$
\begin{aligned}
\dot{x} & =V\{H, x\} \\
& =\left\{H_{m+1}, x\right\}+\left(1+g_{1}\right)\left\{H_{m}, x\right\}+\ldots+\left(1+g_{1}+\ldots+g_{m-1}\right)\left\{H_{2}, x\right\}, \\
\dot{y} & =V\{H, y\} \\
& =\left\{H_{m+1}, y\right\}+\left(1+g_{1}\right)\left\{H_{m}, y\right\}+\ldots+\left(1+g_{1}+\ldots+g_{m-1}\right)\left\{H_{2}, y\right\},
\end{aligned}
$$

and $V$ and $H$ are such that

$$
\begin{aligned}
V & =1+\sum_{j=1}^{\infty} g_{j}, \\
H & =\frac{1}{2}\left(x^{2}+y^{2}\right)+\sum_{j=2}^{\infty} H_{j}=\tau_{1} H_{m+1}+\tau_{2} H_{m}+\ldots+\tau_{m} H_{2} \\
& =\int_{\gamma}\left(\frac{d H_{m+1}}{V}+\frac{\left(1+g_{1}\right) d H_{m}}{V}+\ldots+\frac{\left(1+g_{1}+\ldots+g_{m-1}\right) d H_{2}}{V}\right),
\end{aligned}
$$

where $\gamma$ is an oriented curve (see for instance [17]), $\tau_{j}=\tau_{j}(x, y)$ is a convenient analytic function in the neighborhood of the origin such that $\tau_{j}(0,0)=1$, and $g_{j}=g_{j}(x, y)$ is an arbitrary homogenous polynomial of degree $j$ which we choose in such a way that $V$ is the inverse Reeb integrating factor which satisfies the first 
order partial differential equation

$$
\left\{H_{m+1}, \frac{1}{V}\right\}+\left\{H_{m}, \frac{1+g_{1}}{V}\right\}+\ldots+\left\{H_{2}, \frac{1+g_{1}+\ldots+g_{m-1}}{V}\right\}=0 .
$$

Remark 15 (see formula (44) and the proof of Theorem 13 of [10]). From (16), and (15) the following infinite number of equations must hold

$$
\begin{array}{r}
\left\{H_{m+1}, g_{1}\right\}+\left\{H_{m}, g_{2}\right\}+\ldots+\left\{H_{3}, g_{m-1}\right\}+\left\{H_{2}, g_{m}\right\}=0, \\
\left\{H_{m+1}, g_{1}^{2}-g_{2}\right\}+\left\{H_{m}, g_{1} g_{2}-g_{3}\right\}+\ldots+\left\{H_{3}, g_{1} g_{m-1}-g_{m}\right\}+\left\{H_{2}, g_{1} g_{m}+g_{m+1}\right\}=0,
\end{array}
$$

Consequently

$$
\begin{array}{r}
\left.\int_{0}^{2 \pi}\left(\left\{H_{m+1}, g_{1}\right\}+\left\{H_{m}, g_{2}\right\}+\ldots+\left\{H_{3}, g_{m-1}\right\}\right)\right|_{x=\cos t, y=\sin t} d t=0, \\
\left.\int_{0}^{2 \pi}\left(\left\{H_{m+1}, g_{1}^{2}-g_{2}\right\}+\left\{H_{m}, g_{1} g_{2}-g_{3}\right\}+\ldots+\left\{H_{3}, g_{1} g_{m-1}-g_{m}\right\}\right)\right|_{x=\cos t, y=\sin t} d t=0,
\end{array}
$$

Conditions (17) and (18) are equivalent to the following relations .

$$
\begin{aligned}
\left\{H_{m+j+1}, g_{1}\right\}+\left\{H_{m+j}, g_{2}\right\}+\ldots+\left\{H_{3}, g_{m+j-1}\right\}+\left\{H_{2}, g_{m+j}\right\} & =0 \\
\left.\int_{0}^{2 \pi}\left(\left\{H_{m+j+1}, g_{1}\right\}+\left\{H_{m+j}, g_{2}\right\}+\ldots+\left\{H_{3}, g_{m+j-1}\right\}\right)\right|_{x=\cos t, y=\sin t} d t= & 0,
\end{aligned}
$$

for $j \geq 0$.

Theorem 14 can be applied to determine the Poincaré-Liapunov first integral and Reeb inverse integrating factor for the case when the polynomial differential system is given (see section 8 of [11]. Indeed, given a polynomial vector field $\mathcal{X}$ of degree $m$ with a linear type center at the origin of coordinates, using (14) we determine its first integral $H$ and its Reeb inverse integrating factor. Thus, if in (3) $X=\sum_{j=2}^{m} X_{j}$ and $Y=\sum_{j=2}^{m} Y_{j}$ with $X_{j}$ and $Y_{j}$ homogenous polynomials of degree $j$, from (14) equating the terms of the same degree we get

$$
\begin{aligned}
& \left\{H_{j+1}, x\right\}+g_{1}\left\{H_{j}, x\right\}+\ldots+g_{j-1}\left\{H_{2}, x\right\}=X_{j} \\
& \left\{H_{j+1}, y\right\}+g_{1}\left\{H_{j}, y\right\}+\ldots+g_{j-1}\left\{H_{2}, y\right\}=Y_{j}
\end{aligned}
$$


for $j=2, \ldots, m$. Then

$(20)$

$$
\begin{aligned}
\frac{\partial H_{3}}{\partial y}= & -X_{2}-y g_{1}, \\
\frac{\partial H_{3}}{\partial x}= & Y_{2}-x g_{1}, \\
\frac{\partial H_{4}}{\partial y}= & -X_{3}-g_{1} \frac{\partial H_{3}}{\partial y}-y g_{2}, \\
\frac{\partial H_{4}}{\partial x}= & Y_{3}-g_{1} \frac{\partial H_{3}}{\partial x}-x g_{2} \\
\frac{\partial H_{5}}{\partial y}= & -X_{4}-g_{1} \frac{\partial H_{4}}{\partial y}-g_{2} \frac{\partial H_{3}}{\partial y}-y g_{3}, \\
\frac{\partial H_{5}}{\partial x}= & Y_{4}-g_{1} \frac{\partial H_{4}}{\partial x}-g_{2} \frac{\partial H_{3}}{\partial x}-x g_{3}, \\
\vdots & \vdots \\
\frac{\partial H_{m+1}}{\partial y}= & -X_{m}-g_{1} \frac{\partial H_{m}}{\partial y} \ldots-g_{m-2} \frac{\partial H_{3}}{\partial y}-g_{m-1} \frac{\partial H_{2}}{\partial y}, \\
\frac{\partial H_{m+1}}{\partial x}= & -Y_{m}-g_{1} \frac{\partial H_{m}}{\partial x} \ldots-g_{m-2} \frac{\partial H_{3}}{\partial x}-g_{m-1} \frac{\partial H_{2}}{\partial x} \\
\frac{\partial H_{m+k+1}}{\partial y}= & -g_{1} \frac{\partial H_{m+k}}{\partial y} \ldots-g_{m+k-2} \frac{\partial H_{3}}{\partial y}-g_{m+k-1} \frac{\partial H_{2}}{\partial y}, \\
\frac{\partial H_{m+k+1}}{\partial x}= & -g_{1} \frac{\partial H_{m+k}}{\partial x} \ldots-g_{m+k-2} \frac{\partial H_{3}}{\partial x}-g_{m+k-1} \frac{\partial H_{2}}{\partial x}
\end{aligned}
$$

for $k>0$. From the first two equation of (20) it follows that $g_{1}$ must satisfy the first order partial differential equation

$$
\left\{H_{2}, g_{1}\right\}=\frac{\partial X_{2}}{\partial x}+\frac{\partial Y_{2}}{\partial x},
$$

which in view of Corollary 10 has a unique solution. Substituting $g_{1}$ into the first two equations of (20) and using the Eulers Theorem for homogenous polynomial we obtain

$$
H_{3}=\frac{1}{3}\left(x Y_{2}-y X_{2}-2 g_{1} H_{2}\right) .
$$

We shall determine $g_{2}$ as a solution of the first order partial differential equation

$$
\left\{H_{2}, g_{2}\right\}=\frac{\partial X_{3}}{\partial x}+\frac{\partial Y_{3}}{\partial x}-\left\{H_{3}, g_{1}\right\}
$$

where $g_{1}$ is a solution of (21). Then in view of Corollary 10 we get that under the condition

$$
\left.\int_{0}^{2 \pi}\left(\frac{\partial X_{3}}{\partial x}+\frac{\partial Y_{3}}{\partial x}-\left\{H_{3}, g_{1}\right\}\right)\right|_{x=\cos t, y=\sin t} d t=0
$$


$g_{2}$ exists and has the form $g_{2}(x, y)=\tilde{g}_{2}(x, y)+c H_{2}$ where $c$ is a constant. Hence from the third and fourth equation of (20) we get

$$
H_{4}=\frac{1}{4}\left(x Y_{3}-y X_{3}-3 g_{1} H_{3}-2 g_{2} H_{2}\right)
$$

We shall determine $g_{3}$ as a solution of the first order partial differential equation

$$
\left\{H_{2}, g_{3}\right\}=\frac{\partial X_{4}}{\partial x}+\frac{\partial Y_{4}}{\partial x}-\left\{H_{4}, g_{1}\right\}-\left\{H_{3}, g_{2}\right\}
$$

where $g_{1}, g_{2}$ and $H_{3}, H_{4}$ are solutions of the previous differential equations. Then in view of Corollary 10 we get that there exist an unique solution $g_{3}$. Hence from the fifth and sixth equation of (20) we get

$$
H_{5}=\frac{1}{5}\left(x Y_{4}-y X_{4}-4 g_{1} H_{4}-3 g_{2} H_{3}-2 g_{3} H_{2}\right)
$$

By continuing this process we obtain that if $g_{m-1}$ is a solution of the equation

$$
\left\{H_{2}, g_{m-1}\right\}=\frac{\partial X_{m}}{\partial x}+\frac{\partial Y_{m}}{\partial x}-\left\{H_{m}, g_{1}\right\} \ldots-\left\{H_{3}, g_{m-2}\right\},
$$

which exist if and only if

$$
\left.\int_{0}^{2 \pi}\left(\frac{\partial X_{m}}{\partial x}+\frac{\partial Y_{m}}{\partial x}-\left\{H_{m}, g_{1}\right\} \ldots-\left\{H_{3}, g_{m-2}\right\}\right)\right|_{x=\cos t, y=\sin t} d t=0
$$

where the homogenous $g_{m-2}, \ldots, g_{1}$ are solutions of the previous first order differentia system, then the homogenous polynomial of degree $m+1$ can be calculated as follows

$$
H_{m+1}=-\frac{1}{m+1}\left(x Y_{m}-y X_{m}-m g_{1} H_{m}-\ldots-2 g_{m-1} H_{2}\right)
$$

Finally under the conditions

$$
\begin{aligned}
& \left\{H_{2}, g_{m+k-1}\right\}=-\left\{H_{m+k}, g_{1}\right\}-\left\{H_{m+k-1}, g_{2}\right\} \ldots-\left\{H_{3}, g_{m+k-2}\right\}, \\
& 0=\left.\int_{0}^{2 \pi}\left(-\left\{H_{m+k}, g_{1}\right\}-\left\{H_{m+k-1}, g_{2}\right\} \ldots-\left\{H_{3}, g_{m+k-2}\right\}\right)\right|_{x=\cos t, y=\sin t} d t,
\end{aligned}
$$

we get that

$H_{m+k+1}=-\frac{1}{m+k+1}\left(-(m+k) g_{1} H_{m+k}-(m+k-1) g_{2} H_{m+k-1}-\ldots-2 g_{m+k-1} H_{2}\right)$

for $k>0$. Thus we get the expression of $H$ and of the integrating factor $1+\sum_{j=1}^{\infty} g_{j}$.

Below we need the following results.

Let

$$
x=\kappa_{1} X-\kappa_{2} Y, \quad y=\kappa_{2} X+\kappa_{1} Y,
$$

be a non-degenerated linear transformation, i.e. $\kappa_{1}^{2}+\kappa_{2}^{2} \neq 0$. Then differential system (4) becomes

$$
\begin{aligned}
& \dot{X}=-Y(1+\tilde{\Lambda}(X, Y))+X \tilde{\Omega}(X, Y), \\
& \dot{Y}=X(1+\tilde{\Lambda}(X, Y))+Y \tilde{\Omega}(X, Y),
\end{aligned}
$$


where $\tilde{\Lambda}(X, Y)=\Lambda\left(\kappa_{1} X-\kappa_{2} Y, \kappa_{2} X+\kappa_{1} Y\right)$ and $\tilde{\Omega}(X, Y)=\Omega\left(\kappa_{1} X-\kappa_{2} Y, \kappa_{2} X+\right.$ $\left.\kappa_{1} Y\right)$. Here we say that system $(3)$ is reversible with respect to a straight line $l$ through the origin if it is invariant with respect to reversion about $l$ and a reversion of time $t$ (see for instance [5]). In particular Poincaré's Theorem is applied for the case when $(3)$ is invariant under the transformations $(x, y, t) \longrightarrow(-x, y,-t)$, or $(x, y, t) \longrightarrow(x,-y,-t)$.

In the proof of the results which we give later on we need the Poincare's Theorem (see for instance [14], p.122.)

Theorem 16. The origin of system (3) is a center if the system is reversible.

Since a rotation with respect to the origin of coordinates is a particular transformation of type (32), when a center of system (4) is invariant with respect to a straight line it is not restrictive to assume that such straight line is the $\mathrm{x}$ axis. So the center of system (4) will be invariant by the transformation $(x, y, t) \longrightarrow$ $(-x, y,-t)$ or $(x, y, t) \longrightarrow(x,-y,-t)$. We shall study only the first case, i.e. we shall suppose that the $\Lambda-\Omega$ system is invariant with respect to the transformation $(x, y, t) \longrightarrow(-x, y,-t)$.

The following proposition is easy to prove (see for instance [13]).

Proposition 17. Differential system (33) is invariant under the transformation $(X, Y, t) \longrightarrow(-X, Y,-t)$ if and only if it can be written as

$$
\begin{aligned}
& \dot{X}=-Y\left(1+\Theta_{1}\left(X^{2}, Y\right)\right)+X^{2} \Theta_{2}\left(X^{2}, Y\right), \\
& \dot{Y}=X\left(1+\Theta_{1}\left(X^{2}, Y\right)\right)+X Y \Theta_{2}\left(X^{2}, Y\right),
\end{aligned}
$$

Remark 18. Using the notations of (33) and (34) after some computations we can prove that the following relations

$$
\begin{aligned}
& \tilde{\Lambda}(X, Y)=\sum_{j=1}^{m-1} \tilde{\Lambda}_{j}=\sum_{j=1}^{m-1} \sum_{k+n=j} \lambda_{k n} X^{k} Y^{n}=\Theta_{1}\left(X^{2}, Y\right), \\
& \tilde{\Omega}(X, Y)=\sum_{j=1}^{m-1} \tilde{\Omega}_{j}=\sum_{j=1}^{m-1} \sum_{k+n=j} \omega_{k n} X^{k} Y^{n}=X \Theta_{2}\left(X^{2}, Y\right),
\end{aligned}
$$

hold if and only if

$$
\begin{aligned}
& \lambda_{2 l-1, j}=0 \quad \text { for } \quad l=1,2, \ldots,[m / 2] \quad \text { and } j=0, \ldots, m-2 l, \\
& \omega_{2 l, j}=0 \quad \text { for } \quad l=0,2, \ldots,[(m-1) / 2] \quad \text { and } \quad j=0, \ldots, m-1-2 l,
\end{aligned}
$$

where [] denotes the integer part function. Consequently, $\left[\mathrm{m}^{2} / 4\right]$ coefficients of $\tilde{\Lambda}$ must be zero, and $\left[\left(m^{2}+2 m-3\right) / 4\right]$ coefficients of $\tilde{\Omega}$ must be zero.

Corollary 19. Polynomial differential system (34) can be written as

$$
\begin{aligned}
& \dot{X}=-Y\left(1+\Theta_{1}\left(X^{2}, Y\right)\right)+X\left\{H_{2}, \Phi\right\}=P(X, Y), \\
& \dot{Y}=X\left(1+\Theta_{1}\left(X^{2}, Y\right)\right)+Y\left\{H_{2}, \Phi\right\}=Q(X, Y),
\end{aligned}
$$

where $\Phi=\Phi(x, y)$ is a polynomial of degree at most $m-1$ such that $\left\{H_{2}, \Phi\right\}=$ $X \Theta_{2}\left(X^{2}, Y\right)$. In particular all uniform isochronous centers which after a linear 
change of variables $(x, y) \longrightarrow(X, Y)$ are invariant under the transformations $(X, Y, t) \longrightarrow(-X, Y,-t)$ can be written as

$$
\dot{X}=-Y+X\left\{H_{2}, \Phi\right\}, \quad \dot{Y}=X+Y\left\{H_{2}, \Phi\right\}
$$

Proof. By considering that

$$
\left.\int_{0}^{2 \pi} X \Theta_{2}\left(X^{2}, Y\right)\right|_{x=\cos t, y=\sin t} d t=\int_{0}^{2 \pi} \Theta_{2}\left(1-\sin ^{2} t, \sin t\right) \cos t d t=0 .
$$

By Corollary 10 we get that there exists a polynomial $\Phi$ such that $X \Theta_{2}\left(X^{2}, Y\right)=$ $\left\{H_{2}, \Phi\right\}$. Thus we have the formula (35). Since for uniform isochronous centers $\Theta_{1}\left(X^{2}, Y\right)=0$, the corollary follows.

Proposition 20. Any weak center invariant at the origin of system (35) satisfies that the integral of the divergence on the unit circle is zero.

Proof. The weak center of of system (35) satisfies

$$
\begin{aligned}
\frac{\partial P}{\partial X}+\frac{\partial Q}{\partial Y} & =2\left\{H_{2}, \Phi\right\}+X \frac{\partial\left\{H_{2}, \Phi\right\}}{\partial X}+Y \frac{\partial\left\{H_{2}, \Phi\right\}}{\partial Y}+\left\{H_{2}, \Theta_{1}\right\} \\
& =\left\{H_{2}, 2 \Phi+X \frac{\partial \Phi}{\partial X}+Y \frac{\partial \Phi}{\partial Y}+\Theta_{1}\right\}
\end{aligned}
$$

From Proposition 11 satisfy that

$$
\left.\int_{0}^{2 \pi}\left(\frac{\partial P}{\partial X}+\frac{\partial Q}{\partial Y}\right)\right|_{\substack{X=\cos t \\ Y=\sin t}} d t=0
$$

\section{Proofs of Proposition 3 And 4}

Proof of Proposition 3. Sufficiency: If $\int_{0}^{2 \pi} \Omega_{m-1}(\cos t, \sin t) d t=0$ then (12) holds with

$$
\begin{aligned}
& P:=-y\left(1+(m-2)\left(a_{1} y-a_{2} x\right)\right)+x\left(a_{1} x+a_{2} y+\Omega_{m-1}\right), \\
& Q:=x\left(1+(m-2)\left(a_{1} y-a_{2} x\right)\right)+y\left(a_{1} x+a_{2} y+\Omega_{m-1}\right),
\end{aligned}
$$

because $\Omega_{m-1}$ is a homogenous polynomial of degree $m-1$ and from the Euler Theorem for homogenous polynomial we have

$$
\frac{\partial P}{\partial x}+\frac{\partial Q}{\partial y}=(m+1)\left(a_{1} x+a_{2} y+\Omega_{m-1}\right)
$$

Therefore

$$
\left.\int_{0}^{2 \pi}\left(\frac{\partial P}{\partial x}+\frac{\partial Q}{\partial y}\right)\right|_{\substack{x=\cos t \\ y=\sin t}} d t=0
$$

Then in view of Proposition 12 we get that there exist polynomials $F$ and $G$ of degree $m+1$ and $m-1$ respectively, such that system (7) can be written as

$$
\begin{aligned}
& \dot{x}=P=\{F, x\}+(1+G)\left\{H_{2}, x\right\}, \\
& \dot{x}=Q=\{F, y\}+(1+G)\left\{H_{2}, y\right\} .
\end{aligned}
$$


After some computations we obtain

$$
\begin{aligned}
& \left\{F, H_{2}\right\}=2 H_{2}\left(a_{1} x+a_{2} y+\Omega_{m-1}\right), \\
& \left\{G, H_{2}\right\}=-(m+1)\left(a_{1} x+a_{2} y+\Omega_{m-1}\right) .
\end{aligned}
$$

Therefore $\left\{F+\frac{2 H_{2}}{m+1} G, H_{2}\right\}=0$. Consequently $F=-\frac{2 H_{2}}{m+1} G+p\left(H_{2}\right)$, where

$$
p\left(H_{2}\right)=b_{0}+b_{1} H_{2}+\ldots+b_{[m+1) / 2]} H_{2}^{[(m+1) / 2]}
$$

is an arbitrary polynomial of degree $\leq 2[(m+1) / 2]$, where [ ] denotes the integer part function. Inserting $F$ into the differential system (37) and after some computations we get that

$$
\begin{aligned}
& \dot{x}=P=-\frac{2 H_{2}}{m+1}\{G, x\}+\left(1+p^{\prime}\left(H_{2}\right)+\frac{m-1}{m+1} G\right)\left\{H_{2}, x\right\}, \\
& \dot{y}=Q=-\frac{2 H_{2}}{m+1}\{G, y\}+\left(1+p^{\prime}\left(H_{2}\right)+\frac{m-1}{m+1} G\right)\left\{H_{2}, y\right\},
\end{aligned}
$$

where $p^{\prime}\left(H_{2}\right)=\frac{d p}{d H_{2}}$. In order to have that the linear terms of the previous system be $-y$ and $x$ respectively, we need that $b_{1}=0$, because $G(0,0)=0$. Therefore

$$
\begin{aligned}
\dot{H}_{2} & =x \dot{x}+\dot{y} y=-\frac{2 H_{2}}{m+1}(x\{G, x\}+y\{G, y\})=\frac{2 H_{2}}{m+1}\left\{H_{2}, G\right\} \\
\dot{G} & =\frac{\partial G}{\partial x} \dot{x}+\frac{\partial G}{\partial y} y=\left(1+p^{\prime}\left(H_{2}\right)+\frac{m-1}{m+1} G\right)\left(\frac{\partial G}{\partial x}\left\{H_{2}, x\right\}+\frac{\partial G}{\partial y}\left\{H_{2}, y\right)\right. \\
& =\left\{H_{2}, G\right\}\left(1+\frac{m-1}{m+1} G+p^{\prime}\left(H_{2}\right)\right) .
\end{aligned}
$$

Consequently we have the following linear ordinary differential of first order

$$
\frac{d G}{d H_{2}}-\frac{(m-1) G}{2 H_{2}}=\frac{(m+1)\left(1+p^{\prime}\left(H_{2}\right)\right)}{2 H_{2}}
$$

Thus after the integration we have the existence of a first integral $\mathrm{H}$ given by

$$
\begin{aligned}
H & =\frac{H_{2}}{\left(1+\frac{m-1}{m+1} G(x, y)+(m-1) \Gamma(x, y)+[(m+1) / 2] \Phi\left(H_{2}\right)\right)^{2 /(m-1)}} \\
& =H_{2}(1+O(x, y)),
\end{aligned}
$$

hence the origin is a weak center, where

$$
\Gamma=\frac{2 b_{2}}{m-3} H_{2}+\frac{3 b_{3}}{m-5} H_{2}^{2}+\ldots+\frac{([(m+1) / 2]-1) b_{[(m+1) / 2]-1}}{[(m 1+) / 2]-1-(m+1) / 2} H_{2}^{[(m+1) / 2]-2},
$$

and

$$
\Phi\left(H_{2}\right)= \begin{cases}\frac{b_{[(m+1) / 2]}}{[(m 1+) / 2]-(m+1) / 2} H_{2}^{[(m+1) / 2]-1} & \text { if } m \text { is even } \\ b_{[(m+1) / 2]} H_{2}^{(m-1) / 2} \log H_{2} & \text { if } m \text { is odd. }\end{cases}
$$

We observe that if $m$ is odd then this first integral (38) is non analytic at the origin. 
Necessity: Now we suppose that the origin of system (7) is a center and we must prove that (8) holds. Indeed, if the origin is a center then from Theorem 14 it follows that differential system (7) can be written as (see (14)), where the homogenous polynomials $H_{k}$ and $g_{l}$ for $k=2, \ldots, m+1$ and $l=1, \ldots, m-1$ satisfy

(39)

$$
\begin{aligned}
\left\{H_{m+1}, x\right\}+g_{1}\left\{H_{m}, x\right\} \ldots+g_{m-1}\left\{H_{2}, x\right\} & :=X_{m}=x \Omega_{m-1}, \\
\left\{H_{m+1}, y\right\}+g_{1}\left\{H_{m}, y\right\} \ldots+g_{m-1}\left\{H_{2}, y\right\} & =Y_{m}=y \Omega_{m-1}, \\
\left\{H_{m}, x\right\}+g_{1}\left\{H_{m-1}, x\right\} \ldots+g_{m-2}\left\{H_{2}, x\right\} & =0 \\
\left\{H_{m}, y\right\}+g_{1}\left\{H_{m-1}, y\right\} \ldots+g_{m-2}\left\{H_{2}, y\right\} & =0 \\
\vdots & \vdots \\
\vdots & \vdots \\
\left\{H_{5}, x\right\}+g_{1}\left\{H_{4}, x\right\}+g_{2}\left\{H_{3}, x\right\}+g_{3}\left\{H_{2}, x\right\} & =0 \\
\left\{H_{5}, y\right\}+g_{1}\left\{H_{4}, y\right\}+g_{2}\left\{H_{3}, y\right\}+g_{3}\left\{H_{2}, y\right\} & =0 \\
\left\{H_{4}, x\right\}+g_{1}\left\{H_{3}, x\right\}+g_{2}\left\{H_{2}, x\right\} & =0 \\
\left\{H_{4}, y\right\}+g_{1}\left\{H_{3}, y\right\}+g_{2}\left\{H_{2}, y\right\} & =0 \\
\left\{H_{3}, x\right\}+g_{1}\left\{H_{2}, x\right\} & :=X_{2} \\
\left\{H_{3}, y\right\}+g_{1}\left\{H_{2}, y\right\} & =-y(m-2)\left(a_{1} y-a_{2} x\right)+x\left(a_{1} x+a_{2} y\right), \\
& =x(m-2)\left(a_{1} y-a_{2} x\right)+y\left(a_{1} x+a_{2} y\right) .
\end{aligned}
$$

From the last two equations we obtain (see (21) and (22))

$$
g_{1}=(m+1)\left(a_{1} y-a_{2} x\right), \quad H_{3}=-\frac{2}{m+1} H_{2} g_{1} .
$$

After from the third and fourth equations starting from the end of system (39) we have (see (23) and (25))

$$
g_{2}=\frac{1}{m+1} g_{1}^{2}+c H_{2}, \quad H_{4}=\frac{1}{m+1} g_{1}^{2} H_{2}-\frac{c}{2} H_{2}^{2} .
$$

Later from the fifth and sixth equations starting from the end of system (39) we get

$$
g_{3}=\frac{4}{3(m+1)^{2}} g_{1}^{3}-\frac{2 c}{m+1} g_{1} H_{2}, \quad H_{5}=-\frac{3 m+5}{1(m+1)^{2}} g_{1}^{3} H_{2}+\frac{c}{10(m+1)} g_{1} H_{2}^{2},
$$

and so on, where $c$ is an arbitrary constant. Inserting the previous expressions into the homogenous polynomial $\frac{\partial X_{m}}{\partial x}+\frac{\partial Y_{m}}{\partial y}$ we get

$$
\left\{H_{m}, g_{1}\right\}+\left\{H_{m-1}, g_{2}\right\} \ldots+\left\{H_{2}, g_{m-1}\right\}=(m+1) \Omega_{m-1},
$$

which we obtain from the compatibility conditions of the first two equations of (39). After some computations it follows that $\left\{H_{2}, \Phi\right\}=(m+1) \Omega_{m-1}$, for a convenient polynomial $\Phi$ of degree $m-1$. Consequently in view of Proposition 11 we get that $\int_{0}^{2 \pi} \Omega_{m-1}(\cos t, \sin t) d t=0$. In short the proposition is proved. 
Remark 21. Polynomial differential system (7) under the condition (8) can be written as

$$
\begin{aligned}
\dot{x} & =-y\left(1+(m-2)\left(a_{1} y-a_{2} x\right)\right)+x\left(a_{1} x+a_{2} y+\Omega_{m-1}\right) \\
& =-y\left(1+(m-2)\left(a_{1} y-a_{2} x\right)\right)+x\left\{H_{2}, \Psi\right\}, \\
\dot{y} & \left.=x\left(1+(m-2) a_{1} y-a_{2} x\right)\right)+y\left(a_{1} x+a_{2} y+\Omega_{m-1}\right) \\
& =x\left(1+(m-2)\left(a_{1} y-a_{2} x\right)\right)+y\left\{H_{2}, \Psi\right\},
\end{aligned}
$$

where $\left\{H_{2}, \Psi\right\}=a_{1} x+a_{2} y+\Omega_{m-1}$.

\section{Proofs of Theorem 7}

The proof of Theorem 7 follows from the next propositions.

Proposition 22. A quadratic polynomial differential system

$$
\begin{aligned}
& \dot{x}=-y\left(1+n_{1} x+n_{2} y\right)+x\left(a_{1} x+a_{2} y\right), \\
& \dot{y}=x\left(1+n_{1} x+n_{2} y\right)+y\left(a_{1} x+a_{2} y\right),
\end{aligned}
$$

has a weak center at the origin if and only if

$$
a_{1} n_{1}+a_{2} n_{2}=0 \text {. }
$$

Moreover the quadratic differential system (40) satisfying condition (41), after a linear change of variables $(x, y) \longrightarrow(X, Y)$ it is invariant under the transformations $(X, Y, t) \longrightarrow(-X, Y,-t)$.

Proof. Sufficiency: We suppose that (41) holds and we shall show that then the origin of system (40) is a center. Indeed doing the change of variables given in (32) with $\kappa_{1}^{2}+\kappa_{2}^{2} \neq 0$ to system (??) we obtain the differential system

$$
\begin{aligned}
\dot{X}= & -Y\left(1+\left(\kappa_{1} n_{1}+\kappa_{2} n_{2}\right) X+\left(\kappa_{1} n_{2}-\kappa_{2} n_{1}\right) Y\right) \\
& +X\left(\left(\kappa_{1} a_{1}+\kappa_{2} a_{2}\right) X+\left(\kappa_{1} a_{2}-\kappa_{2} a_{1}\right) Y\right), \\
\dot{Y}= & X\left(1+\left(\kappa_{1} n_{1}+\kappa_{2} n_{2}\right) X+\left(\kappa_{1} n_{2}-\kappa_{2} n_{1}\right) Y\right) \\
& +Y\left(\left(\kappa_{1} a_{1}+\kappa_{2} a_{2}\right) X+\left(\kappa_{1} a_{2}-\kappa_{2} a_{1}\right) Y\right) .
\end{aligned}
$$

This system can be written as (34) if and only if

$$
\kappa_{2} a_{1}-\kappa_{1} a_{2}=0, \quad \kappa_{1} n_{1}+\kappa_{2} n_{2}=0 .
$$

Clearly system (42) has a nonzero solution $\kappa_{1}$ and $\kappa_{2}$ if and only if $a_{1} n_{1}+a_{2} n_{2}=$ 0 . Under condition (42) the quadratic system is

$$
\begin{aligned}
& \dot{X}=-Y\left(1+\left(\kappa_{1} n_{2}-\kappa_{2} n_{1}\right) Y\right)+\left(\kappa_{1} a_{1}+\kappa_{2} a_{2}\right) X^{2}, \\
& \dot{Y}=X\left(1+\left(\kappa_{1} n_{2}-\kappa_{2} n_{1}\right) Y\right)+\left(\kappa_{1} a_{1}+\kappa_{2} a_{2}\right) X Y .
\end{aligned}
$$

This system is invariant under the transformation $(X, Y, t) \longrightarrow(-X, Y,-t)$, so in view of Theorem 16 the origin is a center, which in view of Theorem 2 is a weak center. 
Necessity: We now suppose that system (40) has a center at the origin. From Theorem 14 we get that quadratic differential system (40) can be written as

$$
\begin{aligned}
\dot{x} & =\left\{H_{3}, x\right\}+\left(1+g_{1}\right)\left\{H_{2}, x\right\} \\
& =-y\left(1+n_{1} x+n_{2} y\right)+x\left(a_{1} x+a_{2} y\right):=-y+X_{2}, \\
\dot{y} & =\left\{H_{3}, y\right\}+\left(1+g_{1}\right)\left\{H_{2}, y\right\} \\
& =x\left(1+n_{1} x+n_{2} y\right)+y\left(a_{1} x+a_{2} y\right):=x+Y_{2} .
\end{aligned}
$$

Thus

$$
\begin{aligned}
& \left\{H_{3}, x\right\}+g_{1}\left\{H_{2}, x\right\}=-y\left(n_{1} x+n_{2} y\right)+x\left(a_{1} x+a_{2} y\right):=X_{2}, \\
& \left\{H_{3}, y\right\}+g_{1}\left\{H_{2}, y\right\}=x\left(n_{1} x+n_{2} y\right)+y\left(a_{1} x+a_{2} y\right):=Y_{2} .
\end{aligned}
$$

By determining the homogenous polynomial $g_{1}$ as the unique solution of the equation (see (21))

$$
\left\{H_{2}, g_{1}\right\}=\frac{\partial X_{2}}{\partial x}+\frac{\partial Y_{2}}{\partial x}=\left(n_{2}+3 a_{1}\right) x+\left(3 a_{2}-n_{1}\right) y
$$

we get that

$$
g_{1}=\left(n_{1}-3 a_{2}\right) x+\left(n_{2}+3 a_{1}\right) y \text {. }
$$

In view of homogeneity of $H_{2}$ and $H_{3}$ we obtain that (see (22))

$$
H_{3}=\frac{1}{3}\left(x Y_{2}-y X_{2}-2 g_{1} H_{2}\right)=2 H_{2}\left(a_{2} x-a_{1} y\right) .
$$

On the other hand from conditions (20) since $X_{3}=Y_{3}=0$ we get that

$$
\frac{\partial H_{4}}{\partial y}=-g_{1} \frac{\partial H_{3}}{\partial y}-y g_{2}, \quad \frac{\partial H_{4}}{\partial x}=-g_{1} \frac{\partial H_{3}}{\partial x}-x g_{2}
$$

We shall determine $g_{2}$ as a solution of the first order partial differential equation (see $(23))$

$$
\left\{H_{2}, g_{2}\right\}+\left\{H_{3}, g_{1}\right\}=0
$$

where $g_{1}$ is given in (43). Then in view of Corollary 10 with $V=g_{2}$ and $U=$ $-\left\{H_{3}, g_{1}\right\}$, we get that if $\left.\int_{0}^{2 \pi}\left\{H_{3}, g_{1}\right\}\right|_{x=\cos t, y=\sin t} d t=4 \pi\left(a_{1} n_{1}+a_{2} n_{2}\right)=0$, then $g_{2}$ exists and has the form $g_{2}(x, y)=\tilde{g}_{2}(x, y)+c H_{2}$ where $c$ is a constant. Thus we prove the necessity of the condition (41). In short the proposition is proved.

Proposition 23. A cubic polynomial differential system

$$
\begin{aligned}
\dot{x}= & -y\left(1+\mu\left(a_{2} x-a_{1} y\right)\right) \\
& +x\left(a_{1} x+a_{2} y+a_{3} x^{2}+a_{4} y^{2}+a_{5} x y\right), \\
\dot{y}= & x\left(1+\mu\left(a_{2} x-a_{1} y\right)\right) \\
& +y\left(a_{1} x+a_{2} y+a_{3} x^{2}+a_{4} y^{2}+a_{5} x y\right),
\end{aligned}
$$

has a weak center at the origin if and only if

$$
a_{3}+a_{4}=0, \quad a_{1} a_{2} a_{5}+\left(a_{2}^{2}-a_{1}^{2}\right) a_{4}=0,
$$

Moreover system (44) under condition (45) and $(\mu+1)\left(a_{1}^{2}+a_{2}^{2}\right) \neq 0$, after a linear change of variables $(x, y) \longrightarrow(X, Y)$ it is invariant under the transformations $(X, Y, t) \longrightarrow(-X, Y,-t)$. 
We observe that Proposition 23 when $\mu=0$ provides Theorem 2.2 of the Collins' $[4]$.

Proof. Sufficiency: First of all we observe that cubic differential system (44) under the change of variables (32) becomes

(46)

$$
\begin{aligned}
\dot{X}= & -Y\left(1+\mu\left(\kappa_{1} a_{2}-\kappa_{2} a_{1}\right) X-\mu\left(\kappa_{2} a_{2}+\kappa_{1} a_{1}\right) Y\right) \\
& +\frac{X}{\left(\kappa_{1}^{2}+\kappa_{2}^{2}\right)^{2}}\left(\left(\kappa_{1}^{2}+\kappa_{2}^{2}\right)\left(\left(\kappa_{1} a_{1}+\kappa_{2} a_{2}\right) X+\left(\kappa_{1} a_{2}-\kappa_{2} a_{1}\right) Y\right)\right. \\
& +\left(\kappa_{1}^{2} a_{3}+\kappa_{2}^{2} a_{4}+\kappa_{1} \kappa_{2} a_{5}\right) X^{2} \\
& \left.+\left(\kappa_{2}^{2} a_{3}+\kappa_{1}^{2} a_{4}-\kappa_{1} \kappa_{2} a_{5}\right) Y^{2}+\left(\left(\kappa_{1}^{2}-\kappa_{2}^{2}\right) a_{5}+2 \kappa_{1} \kappa_{2}\left(a_{4}-a_{3}\right)\right) X Y\right), \\
\dot{Y}=\quad & X\left(1+\mu\left(\kappa_{1} a_{1}-\kappa_{2} a_{2}\right) X-\mu\left(\kappa_{1} a_{2}-\kappa_{2} a_{1}\right) Y\right) \\
& +\frac{Y}{\left(\kappa_{1}^{2}+\kappa_{2}^{2}\right)^{2}}\left(\left(\kappa_{1}^{2}+\kappa_{2}^{2}\right)\left(\left(\kappa_{1} a_{1}+\kappa_{2} a_{2}\right) X+\left(\kappa_{1} a_{2}-\kappa_{2} a_{1}\right) Y\right)\right. \\
& +\left(\kappa_{1}^{2} a_{3}+\kappa_{2}^{2} a_{4}+\kappa_{1} \kappa_{2} a_{5}\right) X^{2} \\
& \left.+\left(\kappa_{2}^{2} a_{3}+\kappa_{1}^{2} a_{4}-\kappa_{1} \kappa_{2} a_{5}\right) Y^{2}+\left(\left(\kappa_{1}^{2}-\kappa_{2}^{2}\right) a_{5}+2 \kappa_{1} \kappa_{2}\left(a_{4}-a_{3}\right)\right) X Y\right),
\end{aligned}
$$

is invariant under the transformation $(X, Y, t) \longrightarrow(-X, Y,-t)$ if and only if using Proposition 22 we have

$$
\begin{aligned}
\kappa_{2} a_{1}-\kappa_{1} a_{2} & =0, \\
\kappa_{1}^{2} a_{3}+\kappa_{2}^{2} a_{4}+\kappa_{1} \kappa_{2} a_{5}= & 0, \\
+\kappa_{2}^{2} a_{3}+\kappa_{1}^{2} a_{4}-\kappa_{1} \kappa_{2} a_{5}- & 0 .
\end{aligned}
$$

From these conditions it follows that $\kappa_{1}$ and $\kappa_{2}$ such that $\kappa_{1}^{2}+\kappa_{2}^{2} \neq 0$ if and only if

$$
a_{1} \kappa_{2}-a_{2} \kappa_{1}=0 \quad \text { and } \quad a_{3}+a_{4}=0, \quad k_{1} k_{2} a_{5}+\left(k_{2}^{2}-k_{1}^{2}\right) a_{4}=0 .
$$

We suppose that (45) holds and show that then the origin of system (44) is a center. Clearly if $a_{1}^{2}+a_{2}^{2} \neq 0$, then after the change $x=a_{1} X-a_{2} Y, \quad y=a_{2} X+a_{1} Y$, we get that system (46) coincide with system (34) when $\kappa_{1}=a_{1}$ and $\kappa_{2}=a_{2}$, and consequently (47) becomes (45). Since system (46) is invariant under the change $(X, Y, t) \longrightarrow(-X, Y,-t)$ i.e. is reversible by Poincaré Theorem its origin is a center, and by Theorem 2 it is a weak center. Thus the sufficiency of proposition is proved.

Necessity We suppose that the origin of (44) is a center. We must show that (45) holds. Indeed from Theorem 14 it follows that differential system (44) can be written as

$$
\begin{aligned}
& \left\{H_{4}, x\right\}+\left(1+g_{1}\right)\left\{H_{3}, x\right\}+\left(1+g_{1}+g_{2}\right)\left\{H_{2}, x\right\} \\
= & -y\left(1+\mu\left(a_{2} x-a_{1} y\right)\right)+x\left(a_{1} x+a_{2} y+a_{3} x^{2}+a_{4} y^{2}+a_{5} x y\right), \\
& \left\{H_{4}, y\right\}+\left(1+g_{1}\right)\left\{H_{3}, y\right\}+\left(1+g_{1}+g_{2}\right)\left\{H_{2}, y\right\} \\
= & x\left(1+\mu\left(a_{2} x-a_{1} y\right)\right)+y\left(a_{1} x+a_{2} y+a_{3} x^{2}+a_{4} y^{2}+a_{5} x y\right) .
\end{aligned}
$$


Consequently taking the homogenous parts of these two previous equalities we obtain

(48)

$$
\begin{aligned}
\left\{H_{4}, x\right\}+g_{1}\left\{H_{3}, x\right\}+g_{2}\left\{H_{2}, x\right\} & =-\frac{\partial H_{4}}{\partial y}-g_{1} \frac{\partial H_{3}}{\partial y}-g_{2} \frac{\partial H_{2}}{\partial y} \\
& =x\left(a_{3} x^{2}+a_{4} y^{2}+a_{5} x y\right):=x \Omega_{2}=X_{3}, \\
\left\{H_{4}, y\right\}+g_{1}\left\{H_{3}, y\right\}+g_{2}\left\{H_{2}, y\right\} & =\frac{\partial H_{4}}{\partial x}+g_{1} \frac{\partial H_{3}}{\partial x}+g_{2} \frac{\partial H_{2}}{\partial x} \\
& =y\left(a_{3} x^{2}+a_{4} y^{2}+a_{5} x y\right):=y \Omega_{2}=Y_{3}, \\
\left\{H_{3}, x\right\}+g_{1}\left\{H_{2}, x\right\} & =-\frac{\partial H_{3}}{\partial y}-g_{1} \frac{\partial H_{2}}{\partial y} \\
& =-\mu y\left(a_{2} x-a_{1} y\right)+x\left(a_{1} x+a_{2} y\right), \\
\left\{H_{3}, y\right\}+g_{1}\left\{H_{2}, y\right\} & =\frac{\partial H_{3}}{\partial x}+g_{1} \frac{\partial H_{2}}{\partial x} \\
& =\mu x\left(a_{2} x-a_{1} y\right)+y\left(a_{1} x+a_{2} y\right) .
\end{aligned}
$$

From the last two equations we have that (see (21) and (22)))

$$
g_{1}=(\mu-3)\left(a_{2} x-a_{1} y\right), \quad \text { and } \quad H_{3}=2\left(a_{2} x-a_{1} y\right) H_{2}:=\Upsilon_{1} H_{2} .
$$

Inserting $g_{1}$ and $H_{3}$ into the two first equations and taking into account the Euler Theorem for homogenous polynomials we get that $\frac{\partial X_{3}}{\partial x}+\frac{\partial Y_{3}}{\partial x}=4 \Omega_{2}$ consequently (see (23) and (24))

$$
\left\{H_{2}, g_{2}\right\}+\left\{H_{3}, g_{1}\right\}-4 \Omega_{2}=0,
$$

and this equation has solution if and only if (see Corollary 10)

$$
\left.\int_{0}^{2 \pi}\left(\left\{H_{3}, g_{1}\right\}-4 \Omega_{2}\right)\right|_{x=\cos t, y=\sin t} d t=a_{3}+a_{4}=0,
$$

Hence the first two equations of system (48) have a solution if and only if $a_{3}+a_{4}=0$. The solutions of (48) are

$$
\begin{aligned}
H_{4}= & \left(9 a_{2}^{2}-3 a_{2}^{2} \mu-c\right) x^{2}+\left(4 a_{1} a_{2} \mu+4 a_{4}-12 a_{1} a_{2}\right) y x \\
& \left.+\left(6 a_{1}^{2}+\left(2 a_{1}^{2}+a_{2}^{2}\right) \mu+3 a_{2}^{2}-2 a_{2} a_{5}-c\right) y^{2}\right) H_{2} / 2:=\Upsilon_{2} H_{2}, \\
g_{2}= & \alpha_{1} x^{2}+\left(-6 a_{1} a_{2}+2 a_{1} \mu-4 a_{4}\right) y x \\
& +\left(\left(a_{2}^{2}-a_{1}^{2}\right)(\mu-3)+2 a_{5}+\alpha_{1}\right) y^{2}
\end{aligned}
$$

where $c$ is an arbitrary constant. By inserting $a_{3}+a_{4}=0$ into (44) we get

$$
\begin{aligned}
& \dot{x}=-y\left(1-\mu\left(a_{1} y-a_{2} x\right)\right)+x\left(a_{1} x+a_{2} y-a_{4} x^{2}+a_{4} y^{2}+a_{5} x y\right), \\
& \dot{y}=x\left(1-\mu\left(a_{2} x-a_{1} y\right)\right)+y\left(a_{1} x+a_{2} y-a_{4} x^{2}+a_{4} y^{2}+a_{5} x y\right),
\end{aligned}
$$

By calculating $g_{3}$ as the unique polynomial homogenous of degree four solution of the equation $\left\{H_{4}, g_{1}\right\}+\left\{H_{3}, g_{2}\right\}+\left\{H_{2}, g_{3}\right\}=0$, (see (26)) and determining $H_{5}$ from (26) with $X_{4}=Y_{4}=0$ we get $H_{5}=-4 g_{1} H_{4} / 5-3 g_{2} H_{3} / 5-2 g_{3} H_{2} / 5$. From equation

$$
\left\{H_{5}, g_{1}\right\}+\left\{H_{4}, g_{2}\right\}+\left\{H_{3}, g_{3}\right\}+\left\{H_{2}, g_{4}\right\}=0,
$$


(see (29) with $m=5$ ), and in view of Proposition 11 we get that this equation has a solution for the homogenous polynomial $g_{4}$ if and only if

$$
\begin{aligned}
0 & =\left.\int_{0}^{2 \pi}\left(\left\{H_{5}, g_{1}\right\}+\left\{H_{4}, g_{2}\right\}+\left\{H_{3}, g_{3}\right\}\right)\right|_{x=\cos t, y=\sin t} \\
& =3 \pi(\mu+1)^{2}\left(a_{4}\left(a_{2}^{2}-a_{1}^{2}\right)+a_{1} a_{2} a_{5}\right)=0 .
\end{aligned}
$$

Thus $a_{4}\left(a_{2}^{2}-a_{1}^{2}\right)+a_{1} a_{2} a_{5}=0$. The case when $\mu+1=0$ and $m=3$ has been studied in Proposition 3.

If $a_{1}=a_{2}=0$ then system (49) becomes

$$
\begin{aligned}
& \dot{x}=-y+x\left(-a_{4} x^{2}+a_{4} y^{2}+a_{5} x y\right):=-y+x \Omega_{2}, \\
& \dot{y}=x+y\left(-a_{4} x^{2}+a_{4} y^{2}+a_{5} x y\right)=x+y \Omega_{2},
\end{aligned}
$$

which is a polynomial differential system with homogenous nonlinearities. Consequently we have that

$$
\left.\int_{0}^{2 \pi} \Omega_{2}\right|_{x=\cos t, y=\sin t} d t=\left.\int_{0}^{2 \pi}\left(-a_{4} x^{2}+a_{4} y^{2}+a_{5} x y\right)\right|_{x=\cos t, y=\sin t} d t=0 .
$$

Hence and in view of Corollary 4 we obtain that the origin is a weak center. In short the proposition is proved.

Proposition 24. A quartic polynomial differential system

$$
\begin{aligned}
\dot{x}= & -y\left(1+\mu\left(a_{2} x-a_{1} y\right)\right) \\
& +x\left(a_{1} x+a_{2} y+a_{6} x^{3}+a_{7} y^{3}+a_{8} x^{2} y+a_{9} x y^{2}\right), \\
\dot{y}= & x\left(1+\mu\left(a_{2} x-a_{1} y\right)\right) \\
& +y\left(a_{1} x+a_{2} y+a_{6} x^{3}+a_{7} y^{3}+a_{8} x^{2} y+a_{9} x y^{2}\right),
\end{aligned}
$$

has a weak center at the origin under the assumption

(a) $a_{1}^{2}+a_{2}^{2} \neq 0$ if and only if

(i) $a_{1} a_{2} \neq 0$ and

$$
\begin{aligned}
a_{6}+\frac{1}{2 a_{2}^{3}}\left(a_{7}\left(a_{1}^{3}-3 a_{2}^{2} a_{1}\right)+a_{9}\left(a_{2}^{3}-a_{1}^{2} a_{2}\right)\right) & =0, \\
a_{8}+\frac{1}{2 a_{2}^{2} a_{1}}\left(a_{7}\left(3 a_{1}^{3}-3 a_{1} a_{2}^{2}\right)+a_{9}\left(a_{2}^{3}-3 a_{1}^{2} a_{2}\right)\right) & =0 .
\end{aligned}
$$

(ii) $a_{7}=a_{8}=0$ when $a_{2}=0$ and $a_{1} \neq 0$,

(iii) $a_{6}=a_{9}=0$ when $a_{1}=0$ and $a_{2} \neq 0$.

(b) If $a_{1}=a_{2}=0$.

(c) If $\mu+2=0$.

Moreover if we assume that $(\mu+2)\left(a_{1}^{2}+a_{2}^{2}\right) \neq 0$ then system (50) under the conditions $(i),($ ii) and after a linear change of variables $(x, y) \longrightarrow(X, Y)$ it is invariant under the transformations $(X, Y, t) \longrightarrow(-X, Y,-t)$, and in the case (iii) it is invariant under the transformations $(X, Y, t) \longrightarrow(X,-Y,-t)$,

Proof. Sufficiency: Doing the change of variables (32) system (50) can be written as differential system (33) with $m=4$ and

$$
\Lambda:=\mu\left(a_{2} x-a_{1} y\right), \quad \text { and } \quad \Omega:=a_{1} x+a_{2} y+a_{6} x^{3}+a_{7} y^{3}+a_{8} x^{2} y+a_{9} x y^{2},
$$


which is invariant under the transformation $(X, Y, t) \longrightarrow(-X, Y,-t)$ if and only if

(i) $\kappa_{1} \kappa_{2} \neq 0$ and

$$
\begin{aligned}
\kappa_{2} a_{1}-\kappa_{1} a_{2} & =0, \\
a_{6}+\frac{1}{2 \kappa_{2}^{3}}\left(a_{7}\left(\kappa_{1}^{3}-3 \kappa_{2}^{2} \kappa_{1}\right)+a_{9}\left(\kappa_{2}^{3}-\kappa_{1}^{2} \kappa_{2}\right)\right) & =0, \\
a_{8}+\frac{1}{2 \kappa_{2}^{2} \kappa_{1}}\left(a_{7}\left(3 \kappa_{1}^{3}-3 \kappa_{1} \kappa_{2}^{2}\right)+a_{9}\left(\kappa_{2}^{3}-3 \kappa_{1}^{2} \kappa_{2}\right)\right) & =0-
\end{aligned}
$$

(ii) $\kappa_{1}=0 \quad a_{2} \neq 0$, and $a_{1}=a_{6}=a_{9}=0$.

(iii) $\kappa_{2}=0 \quad a_{1} \neq 0$ and $a_{2}=a_{7}=a_{8}=0$.

We suppose that statement (i), (ii) or (iii) hold and we will show that then the origin is a center of system (50). Indeed, if $a_{1} a_{2} \neq 0$, then after the change $x=$ $a_{1} X-a_{2} Y, \quad y=a_{2} X+a_{1} Y$, we get that system (50) coincide with system (34) for $m=4$ and with $\kappa_{1}=a_{1}$ and $\kappa_{2}=a_{2}$ and consequently by considering that this system is invariant under the change $(X, Y, t) \longrightarrow(-X, Y,-t)$ or $(X, Y, t) \longrightarrow$ $(X,-Y,-t)$, i.e. it is reversible.

If $a_{2}=a_{7}=a_{8}=0$ and $a_{1} \neq 0$, then differential equations (50) become

$$
\begin{aligned}
& \left.\dot{x}=-y\left(1-\mu a_{1} y\right)\right)+x^{2}\left(a_{1}+a_{6} x^{2}+a_{9} y^{2}\right), \\
& \left.\dot{y}=x\left(1-\mu a_{1} y\right)\right)+y x\left(a_{1}+a_{6} x^{2}++a_{9} y^{2}\right) .
\end{aligned}
$$

This system is invariant under the change $(x, y, t) \longrightarrow(-x, y,-t)$ i.e. it is reversible.

If $a_{1}=a_{6}=a_{9}=0$ and $a_{2} \neq 0$, then differential system (50) becomes

$$
\begin{aligned}
& \left.\dot{x}=-y\left(1+\mu a_{2} x\right)\right)+x y\left(a_{2}+a_{7} y^{2}+a_{8} x^{2}\right), \\
& \left.\dot{y}=x\left(1+\mu a_{2} x\right)\right)+y^{2}\left(a_{2}+a_{7} y^{2}+a_{8} x^{2}\right) .
\end{aligned}
$$

this system is invariant under the change $(x, y, t) \longrightarrow(x,-y,-t)$ i.e. it is reversible.

Thus in view of the Poincaré Theorem (see Theorem 16) we get that in cases (i), (ii) and (iii) the origin is a center. Furthermore by Theorem 2 this center is a weak center.

Necessity: We prove the necessity of the statement (a). We suppose that the origin is a weak center. Indeed, from Theorem 14 it follows that differential system (50) can be written as

$$
\begin{aligned}
& \left\{H_{5}, x\right\}+\left(1+g_{1}\right)\left\{H_{4}, x\right\}+\left(1+g_{1}+g_{2}\right)\left\{H_{3}, x\right\}+\left(1+g_{1}+g_{2}+g_{3}\right)\left\{H_{2}, x\right\} \\
= & -y\left(1+\mu\left(a_{2} x-a_{1} y\right)\right)+x\left(a_{1} x+a_{2} y+a_{6} x^{3}+a_{7} y^{3}+a_{8} x^{2} y+a_{9} x y^{2}\right), \\
& \left\{H_{5}, y\right\}+\left(1+g_{1}\right)\left\{H_{4}, y\right\}+\left(1+g_{1}+g_{2}\right)\left\{H_{3}, y\right\}+\left(1+g_{1}+g_{2}+g_{3}\right)\left\{H_{2}, y\right\}, \\
= & x\left(1+\mu\left(a_{2} x-a_{1} y\right)\right)+y\left(a_{1} x+a_{2} y+a_{6} x^{3}+a_{7} y^{3}+a_{8} x^{2} y+a_{9} x y^{2}\right) .
\end{aligned}
$$

In view of Corollary 10 and assisted by an algebraic computer we can obtain the solutions of (53), i.e. the homogenous polynomials $H_{5}, H_{3}, g_{1}, g_{3}$ of degree odd are unique and the homogenous polynomials $H_{4}, g_{2}$ of degree even are obtained modulo an arbitrary polynomial of the form $c\left(x^{2}+y^{2}\right)^{k}$ where $k=1,2$. Indeed taking the 
homogenous part of these equations we obtain

$$
\begin{aligned}
& \left\{H_{3}, x\right\}+g_{1}\left\{H_{2}, x\right\}=-y\left(\mu\left(a_{2} x-a_{1} y\right)\right)+x\left(a_{1} x+a_{2} y\right), \\
& \left\{H_{3}, y\right\}+g_{1}\left\{H_{2}, y\right\}=x\left(\mu\left(a_{2} x-a_{1} y\right)\right)+y\left(a_{1} x+a_{2} y\right) .
\end{aligned}
$$

The solutions of these equations are

$$
\begin{gathered}
g_{1}=(\mu-3)\left(a_{2} x-a_{1} y\right), \quad H_{3}=2 H_{2}\left(a_{2} x-a_{1} y\right) . \\
\left\{H_{4}, x\right\}+g_{1}\left\{H_{3}, x\right\}+g_{2}\left\{H_{2}, x\right\}=0, \\
\left\{H_{4}, x\right\}+g_{1}\left\{H_{3}, x\right\}+g_{2}\left\{H_{2}, x\right\}=0,
\end{gathered}
$$

the compatibility condition of these two last equations becomes of $\left\{H_{3}, g_{1}\right\}+$ $\left\{H_{2}, g_{2}\right\}=0$, and since $\left\{H_{3}, g_{1}\right\}=\left\{H_{2},-(3-\mu)\left(a_{2} x-a_{1} y\right)^{2}\right\}$ we get that $\left\{H_{2}, g_{2}-(3-\mu)\left(a_{2} x-a_{1} y\right)^{2}\right\}=0$. Therefore $g_{2}=(3-\mu)\left(a_{2} x-a 1 y\right)^{2}+c_{2} H_{2}$, where $c_{2}$ is a constant. Then from system (54) we obtain the solution

$$
H_{4}=\frac{H_{2}}{2}\left((\mu-3)\left(\left(a_{1}^{2}-a_{2}^{2}\right)\left(x^{2}-y^{2}\right)+4 a_{1} a_{2} x y\right)+c_{1} H_{2}^{2},\right.
$$

where $c_{1}$ is a constant. Inserting these previous solutions $g_{1}, H_{3}, g_{2}$ and $H_{4}$ into the partial differential equations

$$
\begin{aligned}
& \left\{H_{5}, x\right\}+g_{1}\left\{H_{4}, x\right\}+g_{2}\left\{H_{3}, x\right\}+g_{3}\left\{H_{2}, x\right\} \\
= & x\left(a_{6} x^{3}+a_{7} y^{3}+a_{8} x^{2} y+a_{9} x y^{2}\right)=x \Omega_{3}:=X_{4}, \\
& \left\{H_{5}, y\right\}+g_{1}\left\{H_{4}, y\right\}+g_{2}\left\{H_{3}, y\right\}+g_{3}\left\{H_{2}, y\right\} \\
= & y\left(a_{6} x^{3}+a_{7} y^{3}+a_{8} x^{2} y+a_{9} x y^{2}\right)=y \Omega_{3}:=Y_{4}
\end{aligned}
$$

we get that these differential equations have a unique solution. Indeed, in this case (26) becomes

$$
\left\{H_{4}, g_{1}\right\}+\left\{H_{3}, g_{2}\right\}+\left\{H_{2}, g_{3}\right\}=5 \Omega_{3},
$$

because $\frac{\partial X_{4}}{\partial x}+\frac{\partial Y_{4}}{\partial x}=5 \Omega_{3}$, here we have taking into account that $\Omega_{3}$ is a homogenous polynomial of degree 3 . Consequently there exist a unique solution

$$
\begin{aligned}
g_{3}= & \left(a_{1}\left(a_{2}^{2}+2 a_{1}^{2} / 3\right) \mu^{2}-a_{1}\left(10 a_{1}^{2} / 3+4 a_{2}^{2}\right) \mu+a_{1}\left(4 a_{1}^{2}+3 a_{3}^{2}\right)+5\left(a_{9}+2 a_{6}\right) / 3\right) y^{3} \\
& \left(-a_{2}\left(a_{1}^{2}+2 a_{2}^{2} / 3\right) \mu^{2}+a_{2}\left(10 a_{2}^{2} / 3+4 a_{1}^{2}\right) \mu-\left(3 a_{1}^{2}+4 a_{2}^{2}\right) a_{2}-5 / 3\left(a_{8}+2 a_{7}\right)\right) x^{3} \\
& +\left(a_{1}^{3} \mu^{2}-2\left(2 a_{1}^{2}+a_{2}^{2}\right) a_{2} \mu+3 a_{1}\left(a_{1}^{2}+2 a_{2}^{2}\right)+7 a_{6}\right) x^{2} y \\
& +\left(-a_{2}^{3} \mu^{2}+2\left(2 a_{2}^{2}+a_{1}^{2}\right) a_{2} \mu-3 a_{2}\left(a_{1}^{2}+2 a_{2}^{2}\right)-5 a_{7}\right) x y^{2} \\
& +c(1-\mu)\left(a_{1} x+a_{2} y\right) H_{2}
\end{aligned}
$$

of (56) and substituting $g_{3}$ into (55) we get

$$
\begin{aligned}
H_{5}= & -2 / 3 H_{2}\left(\left(-a_{2}^{3} \mu^{2}+a_{2}\left(2 a_{2}^{2}-3 a_{1}^{2}\right) \mu+3 a_{2}\left(3 a_{1}^{2}+a_{2}^{2}\right)-a_{8}-2 a_{7}\right) x^{3}\right. \\
& -\left(a_{1}^{3} \mu^{2}+a_{1}\left(2 a_{1}^{2}-3 a_{2}^{2}\right) \mu+3 a_{1}\left(3 a_{2}^{2}+a_{1}^{2}\right)+a_{9}+2 a_{6}\right) y^{3} \\
& \left(3 a_{1} a_{2}^{2} \mu^{2}+3 a_{1}\left(a_{1}^{2}-4 a_{2}^{2}\right) \mu+9 a_{1}\left(a_{2}^{2}-a_{1}^{2}\right)+3 a_{6}\right) y x^{2} \\
& -\left(3 a_{2} a_{1}^{2} \mu^{2}+3 a_{2}\left(a_{1}^{2}-4 a_{2}^{2}\right) \mu+9 a_{2}\left(a_{2}^{2}-a_{1}^{2}\right)-3 a_{7}\right) y^{2} x \\
& c H_{2}^{2}\left(3 a_{1} y-2 a_{2} x\right),
\end{aligned}
$$

where $c$ is a constant. 
On the other hand from (19) for $m=4$ and $j=0$ we get that

$$
\begin{aligned}
& \left.\int_{0}^{2 \pi}\left(\left\{H_{5}, g_{1}\right\}+\left\{H_{4}, g_{2}\right\}+\left\{H_{3}, g_{3}\right\}\right)\right|_{x=\cos t, y=\sin t} d t \\
& =-\frac{3 \pi(\mu-3)}{3}\left(a_{1}\left(3 a_{7}+a_{8}\right)-a_{2}\left(3 a_{6}+a_{9}\right)\right)=0 .
\end{aligned}
$$

Hence we get that

(i) If $a_{1} a_{2} \neq 0$ then by introducing the notations

$$
\begin{aligned}
& \lambda_{2}=a_{6}-\frac{1}{2 a_{2}^{3}}\left(a_{7}\left(a_{1}^{3}-3 a_{2}^{2} a_{1}\right)+a_{9}\left(a_{2}^{3}-a_{1}^{2} a_{2}\right)\right), \\
& \lambda_{3}=a_{8}-\frac{1}{2 a_{2}^{2} a_{1}}\left(a_{7}\left(3 a_{1}^{3}-3 a_{1} a_{2}^{2}\right)+a_{9}\left(a_{2}^{3}-3 a_{1}^{2} a_{2}\right)\right),
\end{aligned}
$$

we get that

$$
-\frac{3 \pi(\mu-3)}{3}\left(3 a_{2} \lambda_{2}-a_{1} \lambda 3\right)=0 .
$$

(ii) If $a_{1}=0$ and $a_{2} \neq 0$ then $3 a_{7}+a_{8}=0$.

(iii) If $a_{2}=0$ and $a_{1} \neq 0$ then $3 a_{6}+a_{9}=0$.

Again from (28) with $m=5$ we have the differential equation

$$
\left\{H_{5}, g_{1}\right\}+\left\{H_{4}, g_{2}\right\}+\left\{H_{3}, g_{3}\right\}+\left\{H_{2}, g_{4}\right\}=0,
$$

From this equation we get the homogenous polynomial of degree four $g_{4}$ which in view of Corollary 10 can be obtained with an arbitrary term of the form $c\left(x^{2}+y^{2}\right)^{2}$, i.e. $g_{4}$ is equal to

$$
\begin{aligned}
& \left(\left(-9 a_{1}^{4} a_{2}+11\left(3 a_{7}+a_{8}\right) a_{1}^{2}-a_{2}^{2}\left(3\left(11 a_{7}+a_{8}\right)+54\left(a_{7}+a_{8}\right) a_{2}^{2}\right) x^{4}+\frac{1}{18 a_{2}}(\right.\right. \\
& \left.\left(a^{4}-a_{2}^{4}\right)\left(3 a_{2} \mu^{3}+6 a_{2} \mu^{2}\right)+a_{2}^{4}-a_{2} a_{9}\right) \mu-\left(18\left(3 a_{7}+a_{8}\right) a_{1}^{2}-36 a_{2} a_{1} a_{9}+a_{8}\right) a_{1}^{2} \\
& +\left(3 a _ { 1 } ^ { 2 } a _ { 2 } \left(\left(a_{1}^{2}-3 a_{2}^{2}\right) \mu^{3}+2\left(a_{2}^{2}-a_{1}^{2}\right) \mu^{2}+a_{1}^{2}\left(27 a_{2}^{3}+15 a_{7}+5 a_{8}-9 a_{1}^{2} a_{2}\right)\right.\right. \\
& -2 a_{2}\left(a_{1} a_{9}+9 a_{2} a_{7}\right) \mu+54 a_{2}\left(a_{2} a_{7}-a_{1} a_{9}\right)-a_{2}^{2}\left(3\left(11 a_{7}+a_{8}\right)\right) x^{2} y^{2} \\
& +\left(\frac{2}{3} a_{2} a_{1}^{3}(\mu-2) \mu^{2}+\left(\frac{a_{1}}{3}\left(7 a_{7}+10 a_{8}\right)+\frac{5}{2} a_{2} a_{9}-2 a_{2} a_{1}^{3}\right) \mu-12 a_{1} a_{7}\right) x y^{3} \\
& +\left(\frac{2}{3} a_{2} a_{1}^{3}(\mu-2) \mu^{2}+\left(a_{1}\left(\frac{7 a_{7}}{3}+\frac{4 a_{8}}{3}-2 a_{2}^{3}\right)+\frac{a_{2} a_{9}}{3}\right) \mu\right. \\
& \left.-12 a_{1} a_{7}-4 a_{1} a_{8}+4 a_{2} a_{9}\right) x^{3} y+c\left(x^{2}+y^{2}\right)^{2},
\end{aligned}
$$

if $a_{2} \neq 0$. In analogous form we can obtain the expression of $g_{4}$ when $a_{1} \neq 0$. Now we determine the homogenous polynomial $H_{6}$ from (31) with $m=4$ and $k=1$ we obtain

$$
H_{6}=-\frac{5}{6} g_{1} H_{5}-\frac{4}{6} g_{2} H_{4}-\frac{3}{6} g_{3} H_{3}-\frac{2}{6} g_{4} H_{2} .
$$

Since the integral of the homogenous polynomial of degree 5

$$
\left.\int_{0}^{2 \pi}\left(\left\{H_{6}, g_{1}\right\}+\left\{H_{5}, g_{2}\right\}+\left\{H_{4}, g_{3}\right\}+\left\{H_{3}, g_{4}\right\}\right)\right|_{x=\cos t, y=\sin t} d t \equiv 0,
$$


then we obtain that there is a unique solution for the homogenous polynomial $g_{5}$ of degree 5 of the equation

$$
\left\{H_{6}, g_{1}\right\}+\left\{H_{5}, g_{2}\right\}+\left\{H_{4}, g_{3}\right\}+\left\{H_{3}, g_{4}\right\}+\left\{H_{2}, g_{5}\right\}=0 .
$$

see (30) with $m=4$ and $k=2$.

Calculating the homogenous polynomial $H_{7}$ of degree 7 from (31) with $m=4$ and $k=2$ we get

$$
H_{7}=-\frac{6}{7} g_{1} H_{6}-\frac{5}{7} g_{2} H_{5}-\frac{4}{7} g_{3} H_{4}-\frac{3}{7} g_{4} H_{3}-\frac{2}{7} g_{5} H_{2},
$$

and inserting these polynomials into the integrand of the following homogenous polynomial of degree 6 we have

$$
\left.\int_{0}^{2 \pi}\left(\left\{H_{7}, g_{1}\right\}+\left\{H_{6}, g_{2}\right\}+\left\{H_{4}, g_{3}\right\}+\left\{H_{3}, g_{4}\right\}\right)\right|_{x=\cos t, y=\sin t} d t=I\left(a_{1}, a_{2}, \mu\right),
$$

where $I$ is such that

(iii) if $a_{1}=0$ and $a_{2} \neq 0$ then

$$
I\left(a_{1}, 0, \mu\right)=-\frac{\pi a_{1}^{3}(\mu-3)}{63}\left(\left(231 \mu^{2}+1230 \mu+1776\right) a_{7}+7(5 \mu+12)(\mu+2) a_{9}\right)=0 .
$$

(ii) if $a_{2}=0$ and $a_{1} \neq 0$ then

$$
I\left(0, a_{2}, \mu\right)=\frac{5 \pi a_{2}^{3}(\mu-3)}{189}\left(21(5 \mu+12)(\mu+2) a_{6}+\left(49 \mu^{2}+2 \mu-432\right) a_{9}\right)=0,
$$

(i) If $a_{1} a_{2} \neq 0$ then

$$
\begin{aligned}
I\left(a_{1}, a_{2}, \mu\right)= & \frac{\pi(m-3)}{189}\left(\left(21(5 \mu+12)(\mu+2)\left(3 a_{1}^{2}+5 a_{2}^{2}\right)\right) \lambda_{2}+a_{1}\left(\left(105 a_{1}^{2}+427 a_{2}^{2}\right) \mu^{2}\right.\right. \\
& \left.\left.+\left(426 a_{1}^{2}+2306 a_{2}^{2}\right) \mu+504 a_{1}^{2}+3384 a_{2}^{2}\right) \lambda_{3}\right)=0 .
\end{aligned}
$$

By solving equations (57) and (58) and by considering that the three cases can be represented as a linear system $A \xi=0$, where $\xi=\left(a_{6}, a_{9}\right)^{T}$ in the case (iii), $\xi=\left(a_{7}, a_{8}\right)^{T}$ in the case (ii) and $\xi=\left(\lambda_{2}, \lambda_{3}\right)^{T}$ in the case (i). By considering that the determinant of the matrix $A$ is $|A|=(\mu-3)(\mu+2)\left(21 \mu^{2}+128 \mu+212\right)$. Then under the condition $(\mu-3)(\mu+2) \neq 0$ we obtain the necessity of statement (a) of Proposition 24.

Now we study the case when $\mu=3$. After some computations we get that

$$
\begin{aligned}
& \left.\int_{0}^{2 \pi}\left(\left\{H_{5}, g_{1}\right\}+\left\{H_{4}, g_{2}\right\}+\left\{H_{3}, g_{3}\right\}\right)\right|_{x=\cos t, y=\sin t} d t \\
& =-\frac{15 \pi}{2}\left(a_{1}\left(3 a_{7}+a_{8}\right)-a_{2}\left(3 a_{6}+a_{9}\right)\right)=0 .
\end{aligned}
$$

First we shall study the case when $a_{1}=0$, and $a_{2} \neq 0$. From (57) we get that $3 a_{6}+a_{9}=0$. From (58) we obtain that the integral is identically zero because 
$\mu=3$. On the other hand from the relation

$$
\begin{aligned}
& \int_{0}^{2 \pi}\left(\left\{H_{9}, g_{1}\right\}+\left\{H_{8}, g_{2}\right\}+\left\{H_{7}, g_{3}\right\}+\left\{H_{6}, g_{4}\right\}+\left\{H_{5}, g_{5}\right\}\right. \\
& \left.+\left\{H_{4}, g_{6}\right\}+\left\{H_{3}, g_{7}\right\}+\right)\left.\right|_{x=\cos t, y=\sin t} d t=I\left(a_{1}, a_{2}\right)=0,
\end{aligned}
$$

where $I$ is a convenient function in $a_{1}$ and $a_{2}$. If (iii) $a_{1}=0$ and $a_{2} \neq 0$ then $I\left(0, a_{2}\right)=\left(105 a_{2}^{3} a_{7}-105 a_{2}^{3} a_{8}-864 a_{2}^{2} c\right) a_{6}+\left(995 a_{2}^{3} a_{7}+285 a_{2}^{2} a_{8}-288 a_{2}^{2} c\right) a_{9}=0$. By considering that $a_{9}=-3 a_{6}$ we obtain that $\left(3 a_{7}+a_{8}\right) a_{6}=0$. Thus if $3 a_{7}+a_{8} \neq 0$ then $\mu-3=a_{1}=a_{6}=a_{9}=0$. We observe that if $a_{1}=0$ then $\lambda_{2}=a_{6}$ and $\lambda_{3}=a_{9}$. In analogous form we can study the case when $a_{2}=0$ and $a_{1} \neq 0$.

If $3 a_{7}+a_{8}=3 a_{6}+a_{9}=0$ then we obtain differential system

$$
\begin{aligned}
& \cdot x=-y\left(1+\mu\left(a_{2} x-a_{1} y\right)+x\left(a_{1} x+a_{2} y+a_{6} x\left(x^{2}-3 y^{2}\right)+a_{7} y\left(y^{2}-3 x^{2}\right)\right),\right. \\
& \cdot y=x\left(1+\mu\left(a_{2} x-a_{1} y\right)+y\left(a_{1} x+a_{2} y+a_{6} x\left(x^{2}-3 y^{2}\right)+a_{7} y\left(y^{2}-3 x^{2}\right)\right),\right.
\end{aligned}
$$

From (29) we have that this system has a weak center at the origin if and only if

$$
a_{2}\left(3 a_{1}^{2}-a_{2}^{2}\right) a_{6}+a_{1}\left(a_{1}^{2}-3 a_{2}^{2}\right) a_{7}=0 .
$$

Hence if $a_{1}=0$ then $a_{6}=a_{9}=0$, and if $a_{2}=0$ then $a_{7}=a_{8}=0$. In short we prove that the origin is a weak center of (50) in the case when $\mu=3$. Thus we obtain the necessity of the condition in statement (a). The necessity and sufficiency of statement (c), i.e. when $\mu+2=0$ follows from Proposition 3. Finally we study the necessity and sufficiency of statement (b). Thus when $a_{1}=a_{2}=0$ we obtain that differential system (50) becomes

$$
\begin{aligned}
& \dot{x}=-y+x\left(a_{6} x^{3}+a_{7} y^{3}+a_{8} x y+a_{9} x y^{2}\right):=-y+x \Omega_{3}, \\
& \dot{y}=x+y\left(a_{6} x^{3}+a_{7} y^{3}+a_{8} x y+a_{9} x y^{2}\right):=x+y \Omega_{3},
\end{aligned}
$$

which is a polynomial differential system of degree four with homogenous nonlinearities. Consequently $\left.\int_{0}^{2 \pi} \Omega_{3}\right|_{x=\cos t, y=\sin t} d t=0$, then in view of Proposition 4 we get that the origin is a weak center. In short the proposition is proved.

Proposition 25. A polynomial differential system of degree five

$$
\begin{aligned}
\dot{x}= & -y\left(1+\mu\left(a_{2} x-a_{1} y\right)\right) \\
& +x\left(a_{1} x+a_{2} y+a_{10} x^{4}+a_{11} x^{3} y+a_{12} x^{2} y^{2}+a_{13} x y^{3}+a_{14} y^{4}\right), \\
\dot{y}= & x\left(1+\mu\left(a_{2} x-a_{1} y\right)\right) \\
& +y\left(a_{1} x+a_{2} y++a_{10} x^{4}+a_{11} x^{3} y+a_{12} x^{2} y^{2}+a_{13} x y^{3}+a_{14} y^{4}\right),
\end{aligned}
$$

has a weak center at the origin if and only if

(a)

$$
\begin{aligned}
a_{12}+3\left(a_{10}+a_{14}\right) & =0 \\
2 a_{1}^{3} a_{2}^{3} a_{13}-\left(a_{1}^{6}+7\left(a_{1}^{2} a_{2}^{4}-a_{2}^{4} a_{1}^{2}\right) a_{10}-\left(a_{1}^{5} a_{2}-4 a_{1}^{3} a_{2}^{3}+a_{1} a_{2}^{5}\right) a_{11}=\right. & 0 \\
2 a_{1}^{2} a_{2}^{2} a_{14}-\left(a_{1}^{4}-4 a_{1}^{2} a_{2}^{2}+a_{2}^{4}\right) a_{10}-\left(a_{1}^{3} a_{2}-a_{1} a_{2}^{3}\right) a_{11}= & 0 .
\end{aligned}
$$

Moreover

(i) If $a_{1}=0$ and $a_{2} \neq 0$ then $a_{12}=a_{10}=a_{14}=0$. 
(ii) If $a_{2}=0$ and $a_{1} \neq 0$ then $a_{12}=a_{10}=a_{14}=0$.

(iii) If $a_{1} a_{2} \neq 0$ then by introducing the notations

$$
\begin{aligned}
& \lambda_{1}=a_{13}-\frac{1}{2 a_{1}^{3} a_{2}^{3}}\left(\left(a_{1}^{6}+7\left(a_{1}^{2} a_{2}^{4}-a_{1}^{4} a_{2}^{2}-a_{2}^{6}\right) a_{10}-\left(a_{1}^{5} a_{2}-4 a_{1}^{3} a_{2}^{3}+a_{1} a_{2}^{5}\right) a_{11}\right),\right. \\
& \lambda_{2}=a_{14}-\frac{1}{2 a_{1}^{3} a_{2}^{3}}\left(\left(a_{1}^{4}-4 a_{1}^{2} a_{2}^{2}+a_{2}^{4}\right) a_{10}-\left(a_{1}^{3} a_{2}-a_{1} a_{2}^{3}\right) a_{11}\right), \\
& \text { we get that } \lambda_{1}=\lambda_{2}=0 . \\
& \text { (b) } a_{1}=a_{1}=0, \text { and } a_{12}+3\left(a_{10}+a_{14}\right)=0 . \\
& \text { (c) } \mu+3=0 .
\end{aligned}
$$

Moreover system (59) under the conditions $(60)$ and $(\mu+3)\left(a_{1}^{2}+a_{2}^{2}\right) \neq 0$ and after a linear change of variables $(x, y) \longrightarrow(X, Y)$ it is invariant under the transformations $(X, Y, t) \longrightarrow(-X, Y,-t)$, or $(X, Y, t) \longrightarrow(X,-Y,-t)$.

Proof. Sufficiency: First we write system (59) after the linear change (32). Later we observe that the polynomial differential system obtained is invariant under the transformation $(X, Y, t) \longrightarrow(-X, Y,-t)$ if and only if

$$
\begin{aligned}
& \kappa_{1} a_{2}-\kappa_{2} a_{1}= 0, \\
& 2 \kappa_{1}^{2} \kappa_{2}^{2} a_{12}+\left(3 \kappa_{1}^{4}-6 \kappa_{2}^{2} \kappa_{1}^{2}+3 \kappa_{2}^{4}\right) a_{10}+\left(3 \kappa_{1}^{3} \kappa_{2}-3 \kappa_{2}^{3} \kappa_{1}\right) a_{11}= 0, \\
& 2 \kappa_{1}^{3} \kappa_{2}^{3} a_{13}-\left(\kappa_{1}^{6}-7 \kappa_{1}^{4} \kappa_{2}^{2}+7 \kappa_{1}^{2} \kappa_{2}^{4}-\kappa_{2}^{6}\right) a_{10}-\left(\kappa_{1}^{5} \kappa_{2}-4 \kappa_{2}^{3} \kappa_{1}^{3}+\kappa_{1} \kappa_{2}^{5}\right) a_{11}=0, \\
& 2 \kappa_{1}^{2} \kappa_{2}^{2} a_{14}-\left(\kappa_{1}^{4}-4 \kappa_{1}^{2} \kappa_{2}^{2}+\kappa_{2}^{4}\right) a_{10}+\left(\kappa_{1} \kappa_{2}^{3}-\kappa_{1}^{3} \kappa_{2}\right) a_{11}=0,
\end{aligned}
$$

From the last three conditions it follows that $a_{12}+3\left(a_{10}+a_{14}\right)=0$.

We suppose that (60) holds and then the origin is a center of system (59), and by Theorem 2 is a weak center. We suppose that $a_{1}^{2}+a_{2}^{2} \neq 0$. Then after the change $x=a_{1} X-a_{2} Y, \quad y=a_{2} X+a_{1} Y$, we get that system (59) coincides with system (34) with $\kappa_{1}=a_{1}$ and $\kappa_{2}=a_{2}$, and consequently system (59) is invariant under the change $(X, Y, t) \longrightarrow(-X, Y,-t)$. Thus in view of the Poincaré Theorem we get that the origin is a center. Again by Theorem 2 this center is weak.

We observe that under the assumptions of statement (iii) differential system (59) becomes

$\dot{x}=y\left(1+\mu a_{2} x\right)+x y\left(a_{2}+a_{11} x^{3}+a_{13} x y^{2}\right), \quad \dot{y}=x\left(1+\mu a_{2} x\right)+y^{2}\left(a_{2}+a_{11} x^{3}+a_{13} x y^{2}\right)$.

This system is invariant under the transformation $(x, y, t) \longrightarrow(x,-y,-t)$. Under hypothesis of statement (ii) differential system (59) becomes

$\dot{x}=-y\left(1-\mu a_{1} y\right)+x^{2}\left(a_{1}+a_{11} x^{2} y+a_{13} y^{3}\right), \quad \dot{y}=x\left(1-\mu a_{1} y\right)+y x\left(a_{2}+a_{11} x^{2} y+a_{13} y^{2}\right)$.

This system is invariant under the transformation $(x, y, t) \longrightarrow(-x, y,-t)$. Under the assumptions of statement (i) the conditions $\lambda_{1}=\lambda_{2}=0$ follows immediately.

Necessity Now we suppose that the origin is a center of (59) and we prove that (60) holds. Indeed, from Theorem 14 it follows that differential system (59) can be 
written as

$$
\begin{aligned}
& \left\{H_{6}, x\right\}+\left(1+g_{1}\right)\left\{H_{5}, x\right\}+\left(1+g_{1}+g_{2}\right)\left\{H_{4}, x\right\}+\left(1+g_{1}+g_{2}+g_{3}\right)\left\{H_{3}, x\right\} \\
& +\left(1+g_{1}+g_{2}+g_{3}+g_{4}\right)\left\{H_{2}, x\right\} \\
= & -y\left(1+\mu\left(a_{2} x-a_{1} y\right)\right)+x\left(a_{1} x+a_{2} y+a_{10} x^{4}+a_{11} x^{3} y+a_{12} x^{2} y^{2}+a_{13} x y^{3}+a_{14} y^{4}\right), \\
& \left\{H_{6}, y\right\}+\left(1+g_{1}\right)\left\{H_{5}, y\right\}+\left(1+g_{1}+g_{2}\right)\left\{H_{4}, y\right\}+\left(1+g_{1}+g_{2}+g_{3}\right)\left\{H_{3}, y\right\} \\
& +\left(1+g_{1}+g_{2}+g_{3}+g_{4}\right)\left\{H_{2}, y\right\} \\
= & x\left(1+\mu\left(a_{2} x-a_{1} y\right)\right)+y\left(a_{1} x+a_{2} y++a_{10} x^{4}+a_{11} x^{3} y+a_{12} x^{2} y^{2}+a_{13} x y^{3}+a_{14} y^{4}\right),
\end{aligned}
$$

which is equivalent to

$$
\begin{aligned}
& \left\{H_{3}, x\right\}+g_{1}\left\{H_{2}, x\right\}=-y \mu\left(a_{2} x-a_{1} y\right)+x\left(a_{1} x+a_{2} y\right), \\
& \left\{H_{3}, y\right\}+g_{1}\left\{H_{2}, y\right\}=-y \mu\left(a_{2} x-a_{1} y\right)+y\left(a_{1} x+a_{2} y\right) .
\end{aligned}
$$

These equations have the unique solutions $g_{1}$ and $H_{3}$. Inserting these homogenous polynomials into the equations

$$
\begin{aligned}
& \left\{H_{4}, x\right\}+g_{1}\left\{H_{3}, x\right\}+g_{2}\left\{H_{2}, x\right\}=0, \\
& \left\{H_{4}, y\right\}+g_{1}\left\{H_{3}, y\right\}+g_{2}\left\{H_{2}, y\right\}=0
\end{aligned}
$$

we get $H_{4}$ and $g_{2}$ with arbitrary terms of the form $c_{k}\left(x^{2}+y^{2}\right)^{k}$ with $c_{k}$ a constant for $k=1,2$. Inserting $g_{1}, H_{3}, g_{2}$ and $H_{4}$ into the equations

$$
\begin{aligned}
& \left\{H_{5}, x\right\}+g_{1}\left\{H_{4}, x\right\}+g_{2}\left\{H_{3}, x\right\}+g_{3}\left\{H_{2}, x\right\}=0 \\
& \left\{H_{5}, y\right\}+g_{1}\left\{H_{4}, y\right\}+g_{2}\left\{H_{3}, y\right\}+g_{3}\left\{H_{2}, y\right\}=0
\end{aligned}
$$

we have a unique solutions $g_{3}$ and $H_{5}$. Inserting $g_{j}$ and $H_{j+2}$ for $j=1,2,3$ into the equations

$$
\begin{aligned}
& \left\{H_{6}, x\right\}+g_{1}\left\{H_{5}, x\right\}+g_{2}\left\{H_{4}, x\right\}+g_{3}\left\{H_{3}, x\right\}+g_{4}\left\{H_{2}, x\right\} \\
= & x\left(a_{10} x^{4}+a_{11} x^{3} y+a_{12} x^{2} y^{2}+a_{13} x y^{3}+a_{14} y^{4}\right)=x \Omega_{4}, \\
& \left\{H_{6}, y\right\}+g_{1}\left\{H_{5}, y\right\}+g_{2}\left\{H_{4}, y\right\}+g_{3}\left\{H_{3}, y\right\}+g_{4}\left\{H_{2}, y\right\} \\
= & y\left(a_{10} x^{4}+a_{11} x^{3} y+a_{12} x^{2} y^{2}+a_{13} x y^{3}+a_{14} y^{4}\right)=y \Omega_{4},
\end{aligned}
$$

we get that this partial differential system has solution if and only if and only if $a_{12}+3\left(a_{10}+a_{14}\right)=0$. Indeed from the computability condition

$$
\left\{H_{5}, g_{1}\right\}+\left\{H_{4}, g_{2}\right\}+\left\{H_{3}, g_{3}\right\}+\left\{H_{2}, g_{4}\right\}=6 \Omega_{4},
$$

we obtain that if

$$
\begin{gathered}
\left.\int_{0}^{2 \pi}\left(\left\{H_{5}, g_{1}\right\}+\left\{H_{4}, g_{2}\right\}+\left\{H_{3}, g_{3}\right\}-6 \Omega_{4}\right)\right|_{x=\cos t, y=\sin t} d t \\
=a_{12}+3\left(a_{10}+a_{14}=0 .\right.
\end{gathered}
$$

Then there exist solutions $g_{4}$ and $H_{6}$ which we obtain with arbitrary terms of the form $d_{k}\left(x^{2}+y^{2}\right)^{k}$ with $d_{k}$ a constant for $k=1,2$. 
Inserting the homogenous polynomials $g_{j}$ and $H_{j+2}$ of degree $j$ and $j+2$ respectively, for $j=1,2,3,4$ into (30) for $m=5$ and $k=2$ we get

$$
\begin{gathered}
\left.\int_{0}^{2 \pi}\left(\left\{H_{7}, g_{1}\right\}+\left\{H_{6}, g_{2}\right\}+\left\{H_{5}, g_{3}\right\}+\left\{H_{4}, g_{4}\right\}+\left\{H_{3}, g_{5}\right\}\right)\right|_{x=\cos t, y=\sin t} d t \\
=I_{1}\left(a_{1}, a_{2}\right)=0 .
\end{gathered}
$$

where $I_{1}\left(a_{1}, a_{2}\right)$ is such that

$$
\begin{aligned}
I_{1}\left(a_{1}, 0\right) & =2 \pi a_{1}^{2}(\mu+3)(\mu+2)\left(a_{10}-a_{14}\right)=0, \\
I_{1}\left(0, a_{2}\right) & =2 \pi a_{2}^{2}(\mu+3)(\mu+2)\left(a_{10}-a_{14}\right)=0, \\
\left.I_{1}\left(a_{1}, a_{2}\right)\right|_{a_{1} a_{2} \neq 0} & =2 \pi(\mu+3)(\mu+2)\left(\left(a_{1}^{2}-a_{2}^{2}\right) \lambda_{1}+a_{1} a_{2} \lambda_{2}\right)=0 .
\end{aligned}
$$

and

$$
\begin{aligned}
& \int_{0}^{2 \pi}\left(\left\{H_{9}, g_{1}\right\}+\left\{H_{8}, g_{2}\right\}+\left\{H_{7}, g_{3}\right\}+\left\{H_{6}, g_{4}\right\}+\left\{H_{5}, g_{5}\right\}\right. \\
& \left.+\left\{H_{4}, g_{6}\right\}+\left\{H_{3}, g_{7}\right\}\right)\left.\right|_{x=\cos t, y=\sin t} d t=I_{2}\left(a_{1}, a_{2}\right)=0,
\end{aligned}
$$

where $=I_{2}\left(a_{1}, a_{2}\right)$ is a constant such that

$$
\begin{aligned}
\left.I_{2}\left(a_{1}, 0\right)\right|_{a_{10}=a_{14}} & =-\frac{\pi a_{1}^{4}}{864}\left(2120 \mu^{3}+7161 \mu^{2}+5202 \mu+7857\right) a_{14}=0, \\
\left.I_{2}\left(0, a_{2}\right)\right|_{a_{10}=a_{14}} & =-\frac{\pi a_{2}^{4}}{864}\left(2120 \mu^{3}+7161 \mu^{2}+5202 \mu+7857\right) a_{14}=0=0, \\
\left.I_{2}\left(a_{1}, a_{2}\right)\right|_{a_{1} a_{2} \neq 0} & =\nu_{1} \lambda_{1}+\nu_{2} \lambda_{2}=0 .
\end{aligned}
$$

where $\nu_{1}$ and $\nu_{2}$ are convenient constants such that the system

$$
2 \pi(\mu+3)(\mu+2)\left(\left(a_{1}^{2}-a_{2}^{2}\right) \lambda_{1}+a_{1} a_{2} \lambda_{2}\right):=\sigma_{1} \lambda_{1}+\sigma_{2} \lambda_{2}=0, \quad \nu_{1} \lambda_{1}+\nu_{2} \lambda_{2}=0,
$$

is such that $\Delta:=\sigma_{1} \nu_{2}-\sigma_{2} \nu_{1}=(\mu+3)(\mu+2) p\left(\mu, a_{1}, a_{2}\right)$ with $p\left(\mu, a_{1}, a_{2}\right)$ a convenient polynomial of degree 3 in the variable $\mu$ and $p\left(0, a_{1}, a_{2}\right) \neq 0$.

Consequently if $\mu$ is such that $\Delta \neq 0$ we obtain that $\lambda_{1}=\lambda_{2}=0$ The case when $(\mu+2) p\left(\mu, a_{1}, a_{2}\right)=0$ could be studied in a similar way to the proof of Proposition 24. The proof of statement (c), i.e. when $\mu=-3$ is studied in Proposition 3. Now we prove statement (b). Thus $a_{1}=a_{2}=0$ and the differential system (59) under the condition $a_{12}+3\left(a_{10}+a_{14}\right)=0$ becomes

$$
\begin{aligned}
& \dot{x}=-y+x\left(a_{10} x^{4}+a_{11} x^{3} y-3\left(a_{10}+a_{14}\right) x^{2} y^{2}+a_{13} x y^{3}+a_{14} y^{4}\right), \\
& \dot{y}=x+y\left(a_{10} x^{4}+a_{11} x^{3} y-3\left(a_{10}+a_{14}\right) x^{2} y^{2}+a_{13} x y^{3}+a_{14} y^{4}\right)
\end{aligned}
$$

Hence we get that $\Omega_{4}=a_{10} x^{4}+a_{11} x^{3} y-3\left(a_{10}+a_{14}\right) x^{2} y^{2}+a_{13} x y^{3}+a_{14} y^{4}$ and satisfies the condition $\left.\int_{0}^{2 \pi} \Omega_{4}\right|_{x=\cos t, y=\sin t} d t=0$, because $a_{12}+3\left(a_{10}+a_{14}\right)=$ 0 . Then in view of Corollary 4 we get that the origin is a weak center. Thus the necessity of the statement (b) is proved, and consequently the proposition is proved. 
Proposition 26. The polynomial differential system of degree six (61)

$$
\begin{aligned}
\dot{x}= & -y\left(1+\mu\left(a_{2} x-a_{1} y\right)\right) \\
& +x\left(a_{1} x+a_{2} y+a_{15} x^{5}+a_{16} y^{5}+a_{17} x^{4} y+a_{18} x^{3} y^{2}+a_{19} x^{2} y^{3}+a_{20} x y^{4}\right), \\
\dot{y}= & x\left(1+\mu\left(a_{2} x-a_{1} y\right)\right) \\
& +y\left(a_{1} x+a_{2} y+a_{15} x^{5}+a_{16} y^{5}+a_{17} x^{4} y+a_{18} x^{3} y^{2}+a_{19} x^{2} y^{3}+a_{20} x y^{4}\right),
\end{aligned}
$$

has a weak center at the origin if and only if

(a) the following conditions hold:

$$
\begin{aligned}
& 8 a_{1}^{2} a_{2}^{5} a_{15}+\left(2 a_{1}^{5} a_{2}^{2}-4 a_{1}^{3} a_{2}^{4}+2 a_{1} a_{2}^{6}\right) a_{19}-\left(3 a_{1}^{7}-15 a_{1}^{5} a_{2}^{2}+25 a_{1}^{3} a_{2}^{4}-5 a_{1} a_{2}^{6}\right) a_{16} \\
& +\left(a_{2}^{7}+11 a_{1}^{4} a_{2}^{3}-9 a_{1}^{2} a_{2}^{5}-3 a_{1}^{6} a_{2}\right) a_{20}=0, \\
& 8 a_{1}^{3} a_{2}^{4} a_{17}-\left(15 a_{1}^{7}-55 a_{1}^{5} a_{2}^{2}+45 a_{1}^{3} a_{2}^{4}-5 a_{1} a_{2}^{6}\right) a_{16}-\left(10 a_{1}^{5} a_{2}^{2}-12 a_{1}^{3} a_{2}^{4}+2 a_{1} a_{2}^{6}\right) a_{19} \\
& -\left(-15 a_{1}^{6} a_{2}+35 a_{1}^{4} a_{2}^{3}-13 a_{1}^{2} a_{2}^{5}+a_{2}^{7}\right) a_{20}=0, \\
& 2 a_{1}^{2} a_{2}^{3} a_{18}-\left(5 a_{1}^{5}-10 a_{1}^{3} a_{2}^{2}+5 a_{1} a_{2}^{4}\right) a_{16}-\left(4 a_{1}^{3} a_{2}^{2}-2 a_{1} a_{2}^{4}\right) a_{19}-\left(6 a_{1}^{2} a_{2}^{3}-5 a_{1}^{4} a_{2}-a_{2}^{5}\right) a_{20}=0 .
\end{aligned}
$$

\section{Moreover}

(i) if $a_{1}=0$ and $a_{2} \neq 0$ then $a_{15}=a_{17}=0$,

(ii) if $a_{2}=0$ and $a_{1} \neq 0$ then $a_{16}=a_{18}=0$,

(iii) if $a_{1} a_{2} \neq 0$ then

$$
\begin{aligned}
\lambda_{1}= & a_{15}+\frac{1}{8 a_{1}^{2} a_{2}^{5}}\left(\left(2 a_{1}^{5} a_{2}^{2}-4 a_{1}^{3} a_{2}^{4}+2 a_{1} a_{2}^{6}\right) a_{19}-\left(3 a_{1}^{7}-15 a_{1}^{5} a_{2}^{2}+25 a_{1}^{3} a_{2}^{4}-5 a_{1} a_{2}^{6}\right) a_{16}\right. \\
& \left.+\left(a_{2}^{7}+11 a_{1}^{4} a_{2}^{3}-9 a_{1}^{2} a_{2}^{5}-3 a_{1}^{6} a_{2}\right) a_{20}\right)=0 \\
\lambda_{2}= & a_{17}-\frac{1}{8 a_{1}^{3} a_{2}^{4}}\left(\left(15 a_{1}^{7}-55 a_{1}^{5} a_{2}^{2}+45 a_{1}^{3} a_{2}^{4}-5 a_{1} a_{2}^{6}\right) a_{16}+\left(10 a_{1}^{5} a_{2}^{2}-12 a_{1}^{3} a_{2}^{4}+2 a_{1} a_{2}^{6}\right) a_{19}\right. \\
& \left.+\left(-15 a_{1}^{6} a_{2}+35 a_{1}^{4} a_{2}^{3}-13 a_{1}^{2} a_{2}^{5}+a_{2}^{7}\right) a_{20}\right)=0 \\
\lambda_{3}= & a_{18}+\frac{1}{2 a_{1}^{2} a_{2}^{3}}\left(-\left(5 a_{1}^{5}-10 a_{1}^{3} a_{2}^{2}+5 a_{1} a_{2}^{4}\right) a_{16}\right. \\
& \left.-\left(4 a_{1}^{3} a_{2}^{2}-2 a_{1} a_{2}^{4}\right) a_{19}-\left(6 a_{1}^{2} a_{2}^{3}-5 a_{1}^{4} a_{2}-a_{2}^{5}\right) a_{20}\right)=0 .
\end{aligned}
$$

(b) $a_{1}=a_{2}=0$.

(c) $\mu+4=0$.

Moreover, assume that $(\mu+4)\left(a_{1}^{2}+a_{2}^{2}\right) \neq 0$ then system (61) under the conditions (i), (ii),(iii) and after a linear change of variables $(x, y) \longrightarrow(X, Y)$ it is invariant under the transformations $(X, Y, t) \longrightarrow(-X, Y,-t)$, or $(X, Y, t) \longrightarrow(X,-Y,-t)$.

Proof. Sufficiency: First we observe that the polynomial differential system (61) under the conditions (i) becomes

$$
\begin{aligned}
& \dot{x}=-y\left(1-\mu a_{2} x\right)+x y\left(a_{2}+a_{16} y^{4}+a_{17} x^{4}+a_{19} x^{2} y^{2}\right), \\
& \dot{y}=x\left(1-\mu a_{2} x\right)+y^{2}\left(a_{2}+a_{16} y^{4}+a_{17} x^{4}+a_{19} x^{2} y^{2}\right),
\end{aligned}
$$


which is invariant under the transformation $(x, y, t) \longrightarrow(x,-y,-t)$, and the polynomial differential system (61) under the conditions (ii) becomes

$$
\begin{aligned}
& \dot{x}=-y\left(1+\mu a_{1} y\right)+x^{2}\left(a_{1} x+a_{15} x^{4}+a_{18} x^{2} y^{2}+a_{20} y^{4}\right), \\
& \dot{y}=x\left(1+\mu a_{1} y\right)+x y\left(a_{1} x+a_{15} x^{4}+a_{18} x^{2} y^{2}+a_{20} y^{4}\right),
\end{aligned}
$$

which is invariant under the transformation $(x, y, t) \longrightarrow(-x, y,-t)$

Under the linear change of variables (32) the differential system (61) is invariant under the transformation $(X, Y, t) \longrightarrow(-X, Y,-t)$ if and only if

$$
\begin{aligned}
& \left(2 \kappa_{1}^{5} \kappa_{2}^{2}-4 \kappa_{1}^{3} \kappa_{2}^{4}+2 \kappa_{1} \kappa_{2}^{6}\right) a_{19}-\left(3 \kappa_{1}^{7}-15^{5} \kappa_{1} \kappa_{2}^{2}+25 \kappa_{1}^{3} \kappa_{2}^{4}-5 \kappa_{1} \kappa_{2}^{6}\right) a_{16} \\
& +\left(\kappa_{2}^{7}+11 \kappa_{1}^{4} \kappa_{2}^{3}-9 \kappa_{1}^{2} \kappa_{2}^{5}-3 \kappa_{1}^{6} \kappa_{2}\right) a_{20}+8 \kappa_{1}^{2} \kappa_{2}^{5} a_{15}=0 \\
& \left(15 \kappa_{1}^{7}-55 \kappa_{1}^{5} \kappa_{2}^{2}+45 \kappa_{1}^{3} \kappa_{2}^{4}-5 \kappa_{1} \kappa_{2}^{6}\right) a_{16}+\left(10 \kappa_{1}^{5} \kappa_{2}^{2}-12 \kappa_{1}^{3} \kappa_{2}^{4}+2 \kappa_{1} \kappa_{2}^{6}\right) a_{19} \\
& +\left(-15 a_{1}^{6} a_{2}+35 a_{1}^{4} a_{2}^{3}-13 a_{1}^{2} a_{2}^{5}+a_{2}^{7}\right) a_{20}-8 a_{1}^{3} a_{2}^{4} a_{17}=0 \\
& 2 \kappa_{1}^{2} \kappa_{2}^{3} a_{18}-\left(5 \kappa_{1}^{5}-10 \kappa_{1}^{3} \kappa_{2}^{2}+5 \kappa_{1} \kappa_{2}^{4}\right) a_{16} \\
& -\left(4 \kappa_{1}^{3} \kappa_{2}^{2}-2 \kappa_{1} \kappa_{2}^{4}\right) a_{19}-\left(6 \kappa_{1}^{2} \kappa_{2}^{3}-5 \kappa_{1}^{4} \kappa_{2}-\kappa_{2}^{5}\right) a_{20}=0 .
\end{aligned}
$$

We shall study only the case (iii), i.e. when $a_{1} a_{2} \neq 0$. We suppose that (62) holds and show that then the origin is a center of system (61). For the case when $a_{1}^{2}+a_{2}^{2} \neq 0$, after the change $x=a_{1} X-a_{2} Y, \quad y=a_{2} X+a_{1} Y$, we get that this system coincides with system (35) and with $\kappa_{1}=a_{1}$ and $\kappa_{2}=a_{2}$. Consequently system (35) is invariant under the change $(X, Y, t) \longrightarrow(-X, Y,-t)$. Thus in view of the Poincaré Theorem we get that the origin is a center, and by Theorem 2 this center is a weak center.

Necessity, Now we suppose that the origin is a center of (61) and we shall prove that (62) holds. Indeed, from Theorem 14 it follows that differential system (61) can be written as

$$
\begin{aligned}
& \left\{H_{7}, x\right\}+\left(1+g_{1}\right)\left\{H_{6}, x\right\}+\left(1+g_{1}+g_{2}\right)\left\{H_{5}, x\right\}+\left(1+g_{1}+g_{2}+g_{3}\right)\left\{H_{4}, x\right\} \\
& +\left(1+g_{1}+g_{2}+g_{3}+g_{4}\right)\left\{H_{3}, x\right\}+\left(1+g_{1}+g_{2}+g_{3}+g_{4}+g_{5}\right)\left\{H_{2}, x\right\} \\
= & -y\left(1+\mu\left(a_{2} x-a_{1} y\right)\right)+x\left(a_{1} x+a_{2} y+a_{15} x^{5}+a_{16} y^{5}+a_{17} x^{4} y+a_{18} x^{3} y^{2}\right. \\
& \left.+a_{19} x^{2} y^{3}+a_{20} x y^{4}\right), \\
& \left\{H_{7}, y\right\}+\left(1+g_{1}\right)\left\{H_{6}, y\right\}+\left(1+g_{1}+g_{2}\right)\left\{H_{5}, y\right\}+\left(1+g_{1}+g_{2}+g_{3}\right)\left\{H_{4}, y\right\} \\
& +\left(1+g_{1}+g_{2}+g_{3}+g_{4}\right)\left\{H_{3}, y\right\}+\left(1+g_{1}+g_{2}+g_{3}+g_{4}+g_{5}\right)\left\{H_{2}, y\right\} \\
= & x\left(1+\mu\left(a_{2} x-a_{1} y\right)\right)+y\left(a_{1} x+a_{2} y+a_{15} x^{5}+a_{16} y^{5}+a_{17} x^{4} y+a_{18} x^{3} y^{2}\right. \\
& \left.+a_{19} x^{2} y^{3}+a_{20} x y^{4}\right) .
\end{aligned}
$$


This partial differential system has solution for arbitrary $a_{1}, a_{2}, a_{15}, a_{16}, a_{17}, a_{18}, a_{19}, a_{20}$. After some computations we get that

(63)

$$
\begin{aligned}
\int_{0}^{2 \pi} & \left.\left(\left\{H_{7}, g_{1}\right\}+\left\{H_{6}, g_{2}\right\}+\left\{H_{5}, g_{3}\right\}+\left\{H_{4}, g_{4}\right\}+\left\{H_{3}, g_{5}\right\}\right)\right|_{x=\cos t, y=\sin t} d t \\
& \left.=-\pi(\mu+4)\left(a_{1} \lambda_{2}\left(a_{1}, a_{2}\right)-5 a_{2} \lambda_{1}\left(a_{1}, a_{2}\right)-a_{2} \lambda_{3}\left(a_{1}, a_{2}\right)\right)\right) \\
& :=\nu_{11} \lambda_{1}+\nu_{12} \lambda_{2}+\nu_{13} \lambda_{3}=0
\end{aligned}
$$

where $\lambda_{j}=\lambda_{j}\left(a_{1}, a_{2}, \mu\right)$ for $j=1,2,3$ are constant defined in (62). By continuing the integration of (see equation (30) with $m=6$ and $k=3$ ) we get that

$$
\begin{aligned}
& \int_{0}^{2 \pi}\left(\left\{H_{9}, g_{1}\right\}+\left\{H_{8}, g_{2}\right\}+\left\{H_{7}, g_{3}\right\}+\left\{H_{6}, g_{4}\right\}+\left\{H_{5}, g_{5}\right\}+\left\{H_{4}, g_{6}\right\}\right. \\
& \left.+\left\{H_{3}, g_{7}\right\}\right)\left.\right|_{x=\cos t, y=\sin t} d t:=\nu_{21} \lambda_{1}+\nu_{22} \lambda_{2}+\nu_{23} \lambda_{3}=0,
\end{aligned}
$$

where

$$
\begin{aligned}
\nu_{21}:= & \frac{25 a_{2} \pi}{6912}\left(\left(-1062 a_{1}^{2}-558 a_{2}^{2}\right) \mu^{3}+\left(-8961 a_{1}^{2}-7501 a_{2}^{2}\right) \mu^{2}\right. \\
& \left.+1344 c-8460 a_{1}^{2}-8796 a_{2}^{2}\right) \\
\nu_{22}:= & -\frac{5 a_{1} \pi}{2304}\left(\left(2070 a_{2}^{2}-354 a_{1}^{2}\right) \mu^{3}+\left(13897 a_{2}^{2}-2987 a_{1}^{2}\right) \mu^{2}\right. \\
& +\left(448 c-4308 a_{2}^{2}-2820 a_{1}^{2}\right)+\left(16677 a_{1}^{2}-83367 a_{2}^{2}-1344 c\right), \\
\nu_{23}:= & \frac{5 a_{2}}{6912}\left(4014 a_{1}^{2}-2250 a_{2}^{2}\right) \mu^{3}+\left(27933 a_{1}^{2}-19799 a_{2}^{2}\right) \mu^{2} \\
& \left.+\left(-11556 a_{1}^{2}-7764 a_{2}^{2}+1344 c\right)+99889 a_{2}^{2}-155763 a_{1}^{2}-4032 c\right),
\end{aligned}
$$

where $c$ is an arbitrary constant. Again from equation (30) with $m=6$ and $k=5$ we get

(65)

$$
\begin{aligned}
& \int_{0}^{2 \pi}\left(\left\{H_{11}, g_{1}\right\}+\left\{H_{10}, g_{2}\right\}+\left\{H_{9}, g_{3}\right\}+\left\{H_{8}, g_{4}\right\}+\left\{H_{7}, g_{5}\right\}+\left\{H_{6}, g_{6}\right\}\right. \\
& \left.+\left\{H_{5}, g_{7}\right\}+\left\{H_{4}, g_{8}\right\}\left\{H_{3}, g_{9}\right\}\right)\left.\right|_{x=\cos t, y=\sin t} d t:=\nu_{31} \lambda_{1}+\nu_{32} \lambda_{2}+\nu_{33} \lambda_{3}=0,
\end{aligned}
$$

where $\nu_{3 j}$ for $j=1,2,3$, are convenient constants. From (63), (64) and (65) we obtain the linear system with respect to $\lambda_{1}, \lambda_{2}, \lambda_{3}: A \lambda=0$, where $A=A(\mu)$ is the coefficient matrix with determinant $|A|$ equal to

$$
\begin{aligned}
& \left|\begin{array}{lll}
\nu_{11} & \nu_{12} & \nu_{13} \\
\nu_{21} & \nu_{22} & \nu_{23} \\
\nu_{31} & \nu_{32} & \nu_{33}
\end{array}\right|=-\frac{\pi^{3}\left(a_{2}^{2} a_{1}\right)^{3}(\mu+4)}{456192}\left(12276 \mu^{5}+145467 \mu^{4}+502471 \mu^{3}\right. \\
& \left.\quad+480577 \mu^{2}-3775995 \mu-2701980\right)\left(846 \mu^{3}+6149 \mu^{2}-576 \mu-34299\right) \neq 0 .
\end{aligned}
$$

Consequently if $|A| \neq 0$ then the unique solution of these linear system is the trivial solution $\lambda_{1}=\lambda_{2}=\lambda_{3}=0$. The case when $|A|=0$ could be studied in a similar way of Proposition 24. Thus the necessity of statement (a) is proved. To 
prove statement (c), i.e. when $\mu=-4$ we apply Proposition 3. Finally we prove statement (b), i.e when $a_{1}=a_{2}=0$. Under these conditions system (61) becomes (66)

$$
\begin{aligned}
& \dot{x}=-y+x\left(a_{15} x^{5}+a_{16} y^{5}+a_{17} x^{4} y+a_{18} x^{3} y^{2}+a_{19} x^{2} y^{3}+a_{20} x^{1} y^{4}\right):=-y+x \Omega_{5}, \\
& \dot{y}=x+y\left(a_{15} x^{5}+a_{16} y^{5}+a_{17} x^{4} y+a_{18} x^{3} y^{2}+a_{19} x^{2} y^{3}+a_{20} x^{1} y^{4}:=x+y \Omega_{5},\right.
\end{aligned}
$$

which is a polynomial differential system of degree six with homogenous nonlinearities. Since $\left.\int_{0}^{2 \pi} \Omega_{5}\right|_{x=\cos t, y=\sin t} d t=0$, in view of Proposition 4 we obtain that the origin is a weak center of (66). Thus the necessity of the proposition is proved and consequently the proposition is proved.

\section{Proof of Theorem 9}

The proof of Theorem 9 follows from the next propositions.

Proposition 27. A quadratic polynomial differential system

$$
\begin{aligned}
& \dot{x}=-y\left(1+a_{1} x+a_{2} y\right)+x\left(a_{3} x+a_{4} y\right), \\
& \dot{y}=x\left(1+a_{1} x+a_{2} y\right)+y\left(a_{3} x+a_{4} y\right),
\end{aligned}
$$

has a weak center at the origin if and only if

$$
a_{1} a_{3}+a_{2} a_{4}=0 .
$$

Moreover system (67) under condition (68) and $\left(a_{1}^{2}+a_{2}^{2}\right) \neq 0$ and after a linear change of variables $(x, y) \longrightarrow(X, Y)$ it is invariant under the transformations $(X, Y, t) \longrightarrow(-X, Y,-t)$ or transformations $(X, Y, t) \longrightarrow(X,-Y,-t)$.

Proof. It is analogous to the proof of Proposition 22 with $n_{1}=a_{1}, n_{2}=a_{2}$ and $a_{1}=a_{3}, a_{2}=a_{4}$.

Proposition 28. A cubic polynomial differential system

$$
\begin{aligned}
& \dot{x}=-y\left(1+a_{1} x+a_{2} y\right)+x\left(a_{3} x^{2}+a_{4} y^{2}+a_{5} x y\right), \\
& \dot{y}=x\left(1+a_{1} x+a_{2} y\right)+y\left(a_{3} x^{2}+a_{4} y^{2}+a_{5} x y\right),
\end{aligned}
$$

has a weak center at the origin if and only if

$$
a_{3}+a_{4}=0, \quad a_{1} a_{2} a_{5}+\left(a_{2}^{2}-a_{1}^{2}\right) a_{4}=0,
$$

Moreover system (69), under condition (70) and $a_{1}^{2}+a_{2}^{2} \neq 0$, after a linear change of variables $(x, y) \longrightarrow(X, Y)$ it is invariant under the transformation $(X, Y, t) \longrightarrow$ $(-X, Y,-t)$.

Proof. It is analogous to the proof of Proposition 23.

Proposition 29. A polynomial differential system of degree four

$$
\begin{aligned}
& \dot{x}=-y\left(1+a_{1} x+a_{2} y\right)+x\left(a_{6} x^{3}+a_{7} y^{3}+a_{8} x^{2} y+a_{9} x y^{2}\right), \\
& \dot{y}=x\left(1+a_{1} x+a_{2} y\right)+y\left(a_{6} x^{3}+a_{7} y^{3}+a_{8} x^{2} y+a_{9} x y^{2}\right),
\end{aligned}
$$

has a weak center at the origin under the assumption

(a) $a_{1}^{2}+a_{2}^{2} \neq 0$ if and only if 
(i) $a_{1} a_{2} \neq 0$ and

$$
\begin{aligned}
a_{6}+\frac{1}{2 a_{2}^{3}}\left(a_{7}\left(a_{1}^{3}-3 a_{2}^{2} a_{1}\right)+a_{9}\left(a_{2}^{3}-a_{1}^{2} a_{2}\right)\right) & =0, \\
a_{8}+\frac{1}{2 a_{2}^{2} a_{1}}\left(a_{7}\left(3 a_{1}^{3}-3 a_{1} a_{2}^{2}\right)+a_{9}\left(a_{2}^{3}-3 a_{1}^{2} a_{2}\right)\right) & =0 .
\end{aligned}
$$

(ii) $a_{7}=a_{8}=0$ when $a_{2}=0$ and $a_{1} \neq 0$.

(iii) $a_{6}=a_{9}=0$ when $a_{1}=0$ and $a_{2} \neq 0$.

(b) If $a_{1}=a_{2}=0$.

System (71) under the conditions (i) , (ii) and after a linear change of variables $(x, y) \longrightarrow(X, Y)$ it is invariant under the transformations $(X, Y, t) \longrightarrow(-X, Y,-t)$, and in the case (iii) it is invariant under the transformations $(X, Y, t) \longrightarrow(X,-Y,-t)$,

Proof. It is analogous to the proof of Proposition 24.

Proposition 30. A polynomial differential system of degree five

$$
\begin{aligned}
& \dot{x}=-y\left(1+a_{1} x+a_{2} y\right)+x\left(a_{15} x^{5}+a_{16} y^{5}+a_{17} x^{4} y+a_{18} x^{3} y^{2}+a_{19} x^{2} y^{3}+a_{20} x y^{4}\right), \\
& \dot{y}=x\left(1+a_{1} x+a_{2} y\right)+y\left(a_{15} x^{5}+a_{16} y^{5}+a_{17} x^{4} y+a_{18} x^{3} y^{2}+a_{19} x^{2} y^{3}+a_{20} x y^{4},\right.
\end{aligned}
$$

has a weak center at the origin under the assumption

(a) $a_{1}^{2}+a_{2}^{2} \neq 0$ if and only if

(i) $a_{1} a_{2} \neq 0$ and

$$
\begin{aligned}
& 0= a_{15}+\frac{1}{8 a_{1}^{2} a_{2}^{5}}\left(\left(2 a_{1}^{5} a_{2}^{2}-4 a_{1}^{3} a_{2}^{4}+2 a_{1} a_{2}^{6}\right) a_{19}-\left(3 a_{1}^{7}-15 a_{1}^{5} a_{2}^{2}+25 a_{1}^{3} a_{2}^{4}-5 a_{1} a_{2}^{6}\right) a_{16}\right. \\
&\left.+\left(a_{2}^{7}+11 a_{1}^{4} a_{2}^{3}-9 a_{1}^{2} a_{2}^{5}-3 a_{1}^{6} a_{2}\right) a_{20}\right), \\
& 0= a_{17}-\frac{1}{8 a_{1}^{3} a_{2}^{4}}\left(\left(15 a_{1}^{7}-55 a_{1}^{5} a_{2}^{2}+45 a_{1}^{3} a_{2}^{4}-5 a_{1} a_{2}^{6}\right) a_{16}+\left(10 a_{1}^{5} a_{2}^{2}-12 a_{1}^{3} a_{2}^{4}+2 a_{1} a_{2}^{6}\right) a_{19}\right. \\
&\left.+\left(-15 a_{1}^{6} a_{2}+35 a_{1}^{4} a_{2}^{3}-13 a_{1}^{2} a_{2}^{5}+a_{2}^{7}\right) a_{20}\right), \\
& 0= a_{18}+\frac{1}{2 a_{1}^{2} a_{2}^{3}}\left(-\left(5 a_{1}^{5}-10 a_{1}^{3} a_{2}^{2}+5 a_{1} a_{2}^{4}\right) a_{16}\right. \\
&\left.-\left(4 a_{1}^{3} a_{2}^{2}-2 a_{1} a_{2}^{4}\right) a_{19}-\left(6 a_{1}^{2} a_{2}^{3}-5 a_{1}^{4} a_{2}-a_{2}^{5}\right) a_{20}\right) . \\
& \text { (ii) } a_{1}=a_{16}=a_{17}=a_{19}=0 \text { when } a_{2} \neq 0 . \\
& \text { (iii) } a_{2}=a_{15}=a_{18}=a_{20}=0 \text { when } a_{1} \neq 0 . \\
& \text { (b) } \text { If } a_{1}=a_{2}=0 .
\end{aligned}
$$

Moreover, assume that $a_{1}^{2}+a_{2}^{2} \neq 0$ then system (72) under the conditions ( $\left.i\right)$, (ii), and after a linear change of variables $(x, y) \longrightarrow(X, Y)$ it is invariant under the transformations $(X, Y, t) \longrightarrow(-X, Y,-t)$. If (iii) holds then it is invariant under the transformations $(X, Y, t) \longrightarrow(X,-Y,-t)$.

Proof. It is analogous to the proof of Proposition 25. 
We observe that the main difficulty to prove these results for $m>5$ become from the necessary huge computations.

Acknowledgments. The first author is partially supported by a FEDER-MINECO grant MTM2016-77278-P, a MINECO grant MTM2013-40998-P, and an AGAUR grant number 2014SGR-568. The second author was partly supported by the Spanish Ministry of Education through projects TIN2014-57364-C2-1-R, TSI2007-65406C03-01 "AEGIS".

\section{REFERENCES}

[1] M.A. Alwash And N.G. Lloyd, Non-autonomous equations related to polynomial two dimensional systems, Proc. Roy. Soc. Edinburgh 105 A (1987), 129-152.

[2] G. Belitskit, Smooth equivalence of germs of $C^{\infty}$ of vector fields with one zero or a pair of pure imaginary eigenvalues, Funct. Anal. Appl. 20, No. 4 (1986), 253-259.

[3] I. Bendixson, Sur les courbes définies par des equations differentielles, Acta Math. 24 (1901), $1-88$.

[4] C.B. Coluins, Conditions for a centre in a simple class of cubic systems, Differential and Integral Equations 10 (1997), 333-356.

[5] R. Conti, Centers of planar polynomial systems. a review, Le Matematiche, Vol. LIII, Fasc. II, (1998), 207-240.

[6] H. Dulac, Détermination et integration d' une classe d' equations differentielle ayant pour point singulier un centre, Bull. Sci. Math Sér. 32 (1908), 230-252.

[7] M. Frommer, Die Integralkurven einer gewöhnlichen Differential-gleichung erster Ordnung in der Umgebung rationaler Unbestimmtheitsstellen, Math. Ann. 99 (1928), 222-272.

[8] N. Kopell and L.N. Howard, Plane wave solutions to reaction-diffusion equations, Stud. Appl. Math. 52 (1973), 291-328.

[9] J. Llibre and R. Ramírez, Inverse problems in ordinary differential equations and applications, Progress in Math. 313, Birkhäuser, 2016.

[10] J. Llibre, R. RAmírez AND V. RAmírez, An inverse approach to the center-focus problem for polynomial differential system with homogenous nonlinearities, (2017), http://dx.doi.org/10.1016/j.jde.2017.04.030.

[11] J. Llibre, R. Ramírez and V. Ramírez, An inverse approach to the center problem, preprint, (2017).

[12] M.A. Liapounoff, Problème général de la stabilité du mouvement, Annals of Mathematics Studies 17, Princeton University Press, 1947.

[13] I.G. Malkin, Stability theory of movements, Ed. Nauka, Moscow, 1966 (in Russian).

[14] V.V. Nemytski and V.V Stepanov, Qualitative Theory of Differential Equations, Princeton Univ. Press, 1960.

[15] H. Poincaré, Sur l'intégration des équations différentielles du premier ordre et du premier degré I and II, Rendiconti del circolo matematico di Palermo 5 (1891), 161-191; 11 (1897), 193-239.

[16] G. Reeb, Sur certaines propiétés topologiques des variétés feuilletées, W.T. Wu, G. Reeb (Eds.), Sur les espaces fibrés et les variétés feuilletées, Tome XI, in: Actualités Sci. Indust. vol. 1183, Hermann et Cie, (1952) Paris.

[17] S. Sternberg, Lectures on differential geometry, Prentice Hall, 1964.

1 Departament de Matemàtiques, Universitat Autònoma de Barcelona, 08193 Bellaterra, Barcelona, Catalonia, Spain.

E-mail address: jllibre@mat.uab.cat, valentin.ramirez@e-campus.uab.cat

2 Departament d'Enginyeria Informàtica i Matemàtiques, Universitat Rovira i Virgili, Avinguda dels Països Catalans 26, 43007 Tarragona, Catalonia, Spain.

E-mail address: rafaelorlando.ramirez@urv.cat 